\title{
A deep wide-field sub-mm survey of the Carina Nebula complex ${ }^{\star}$
}

\author{
T. Preibisch ${ }^{1}$, F. Schuller ${ }^{2}$, H. Ohlendorf ${ }^{1}$, S. Pekruhl ${ }^{1}$, K. M. Menten ${ }^{2}$, and H. Zinnecker ${ }^{3}$ \\ 1 Universitäts-Sternwarte München, Ludwig-Maximilians-Universität, Scheinerstr. 1, 81679 München, Germany \\ e-mail: preibisch@usm.uni-muenchen.de \\ 2 Max-Planck-Institut für Radioastronomie, Auf dem Hügel 69, 53121 Bonn, Germany \\ 3 Astrophysikalisches Institut Potsdam, An der Sternwarte 16, 14482 Potsdam, Germany \\ Received 19 July 2010 / Accepted 30 September 2010
}

\section{ABSTRACT}

\begin{abstract}
Context. The Great Nebula in Carina is one of the most massive $\left(M_{*, \text { total }} \gtrsim 25000 M_{\odot}\right)$ star-forming complexes in our Galaxy and contains several stars with (initial) masses exceeding $\approx 100 M_{\odot}$; it is therefore a superb location in which to study the physics of violent massive star-formation and the resulting feedback effects, including cloud dispersal and triggered star-formation.

Aims. We aim to reveal the cold dusty clouds in the Carina Nebula complex, to determine their morphology and masses, and to study the interaction of the luminous massive stars with these clouds.

Methods. We used the Large APEX Bolometer Camera LABOCA at the APEX telescope to map a $1.25^{\circ} \times 1.25^{\circ}\left(\widehat{=} 50 \times 50 \mathrm{pc}^{2}\right)$ region at $870 \mu \mathrm{m}$ with $18^{\prime \prime}$ angular resolution $(=0.2 \mathrm{pc}$ at the distance of the Carina Nebula) and an rms noise level of $\approx 20 \mathrm{mJy} / \mathrm{beam}$. Results. From a comparison to $\mathrm{H} \alpha$ images we infer that about $6 \%$ of the $870 \mu \mathrm{m}$ flux in the observed area is likely free-free emission from the HII region, while about $94 \%$ of the flux is very likely thermal dust emission. The total (dust + gas) mass of all clouds for which our map is sensitive is $\sim 60000 M_{\odot}$, in good agreement with the mass of the compact clouds in this region derived from ${ }^{13} \mathrm{CO}$ line observations. There is a wide range of different cloud morphologies and sizes, from large, massive clouds with several $1000 M_{\odot}$, to small diffuse clouds containing just a few $M_{\odot}$. We generally find good agreement in the cloud morphology seen at $870 \mu \mathrm{m}$ and the Spitzer $8 \mu \mathrm{m}$ emission maps, but also identify a prominent infrared dark cloud. Finally, we construct a radiative transfer model for the Carina Nebula complex that reproduces the observed integrated spectral energy distribution reasonably well.

Conclusions. Our analysis suggests a total gas + dust mass of about $200000 M_{\odot}$ in the investigated area; most of this material is in the form of molecular clouds, but a widely distributed component of (partly) atomic gas, containing up to $\sim 50 \%$ of the total mass, may also be present. Currently, only some $10 \%$ of the gas is in sufficiently dense clouds to be immediately available for future star formation, but this fraction may increase with time owing to the ongoing compression of the strongly irradiated clouds and the expected shockwaves of the imminent supernova explosions.
\end{abstract}

Key words. stars: formation - ISM: clouds - ISM: structure - ISM: individual objects: NGC 3372 - submillimeter: ISM

\section{Introduction}

Most stars in the Galaxy are born in massive star-forming regions (e.g., Briceno et al. 2007). The massive stars profoundly influence their environments by creating $\mathrm{H}$ II regions, generating wind-blown bubbles, and exploding as supernovae. This feedback disperses the natal molecular clouds (e.g., Freyer et al. 2003), thus in principle halting further star formation, but ionization fronts and expanding superbubbles can also compress nearby clouds and may thereby trigger the formation of new generations of stars (e.g., Dale et al. 2005; Dale \& Bonnell 2008; Gritschneder et al. 2009). These processes determine the key outputs from star-formation, such as the stellar mass function, the total star formation efficiency, and the frequency of planetary formation. Detailed observational diagnostics of these processes have been hard to come by, mainly because regions with large populations of massive stars and strong feedback are usually too far away for detailed studies.

At a distance of $2.3 \mathrm{kpc}$, the Carina Nebula (NGC 3372; see, e.g., Smith \& Brooks 2008, for an overview) is the nearest southern region with a large massive stellar population

* Based on data acquired with the Atacama Pathfinder Experiment (APEX). APEX is a collaboration between the Max-Planck-Institut für Radioastronomie, the European Southern Observatory, and the Onsala Space Observatory.
(65 O-type stars; see Smith 2006), including several of the most massive and luminous stars known in our Galaxy. The Carina Nebula complex (CNC hereafter) has a total infrared luminosity of $\sim 10^{7} L_{\odot}$ and a size of about $50 \mathrm{pc}$, corresponding to an extent of $1.25^{\circ}$ on the sky. Most of the massive stars reside in one of several clusters $(\operatorname{Tr} 16, \operatorname{Tr} 14, \operatorname{Tr} 15$; see Trumpler 1930) with ages ranging from $\sim 1$ to several Myr. In the central region around $\eta$ Car and the Tr 16 cluster, the molecular clouds have already been largely dispersed by stellar feedback. In the South Pillars (southeast of $\eta$ Car) the clouds are eroded and shaped by the radiation and winds from $\eta$ Car and $\operatorname{Tr} 16$, giving rise to numerous giant dust pillars, which feature very prominently in the mid-infrared images made with the Spitzer Space Observatory (Smith et al. 2010b). The detection of young stellar objects (Megeath et al. 1996) and a very young cluster (the "Treasure Chest Cluster"; see Smith et al. 2005) embedded within the heads of some of the dust pillars suggests that the formation of a new generation of stars is currently triggered in this area.

Because of its prominence on the sky, the CNC has been the target of numerous observations in almost all wavelength bands from $\gamma$-rays to the radio regime (see Smith \& Brooks 2008 , for a summary). Recent deep large-scale surveys of the CNC have been obtained with the the Hubble Space Telescope (HST) (Smith et al. 2010a), the Spitzer infrared observatory 
(Smith et al. 2010b), the near-infrared camera HAWK-I at the ESO Very Large Telescope (Preibisch et al. 2011a, in prep.), and the Chandra X-ray observatory (Townsley et al. 2011; Preibisch et al. 2011b). All these together will allow us for the first time to identify the young low-mass star population in the complex without the strong confusion problems that plague studies based only on optical and infrared data sets.

However, any really comprehensive investigation of this region clearly also requires information on the cool dust and gas in the (molecular) clouds, and the deeply embedded protostars within these clouds. During the very earliest stages of star formation, these dense gas clumps and cores remain very cold $(10-30 \mathrm{~K})$, and therefore escape detection at near- and mid-infrared wavelengths, even with instruments as sensitive as Spitzer. Only the (sub-) millimeter and radio emission from molecular spectral lines and from cool dust allows for an almost un-hindered, unique view onto the processes in the dense clouds. In order to meaningfully complement the extraordinary quality of the recent HST, Spitzer, Chandra, and HAWK-I data, (sub-)mm observations with high spatial resolution, high sensitivity, and large spatial coverage (at least 1 square-degree) are therefore clearly required.

Until recently, the best existing mm-band data set was the SEST/SIMBA survey of the central region of the Carina Nebula by Brooks et al. (2005). Their map of the $1.2 \mathrm{~mm}$ continuum emission covers an area of $10^{\prime} \times 10^{\prime}$ with a half-power beam width of $24^{\prime \prime}$; with a sensitivity limit of $75 \mathrm{mJy}$ per beam, clumps with masses down to $\sim 5 M_{\odot}$ were detected in this survey. Gomez et al. (2010) recently studied the central $30^{\prime} \times 30^{\prime}$ area of the $\mathrm{CNC}$, covering $\eta$ Car and the Keyhole Nebula, at $870 \mu \mathrm{m}$ with LABOCA at the APEX telescope. At radio wavelengths, the most comprehensive existing data set is a NANTEN survey in several CO lines, covering a $4^{\circ} \times 2^{\circ}$ area with a half-power beam width of $2.7^{\prime}$ (Yonekura et al. 2005). The Mopra ${ }^{12} \mathrm{CO}(1-0)$ data with a 43" beam presented by Brooks et al. (1998) (see also Schneider \& Brooks 2004) provide better spatial resolution, but cover only a small area in the central part of the CNC. There was therefore an obvious need for (sub-)mm-data with high spatial resolution as well as high sensitivity, that cover a considerably larger part of the CNC.

In this paper we present the results of our large-scale sub-mm mapping of the CNC with LABOCA at the APEX telescope. Our map covers a $\sim 6$ times larger area and is about twice as sensitive as the sub-mm data from Gomez et al. (2010). After the description of the observations and data analysis (Sect. 2), we discuss general aspects (Sect. 3), describe the structure and properties of the clouds in different regions within the complex (Sect. 4) and compare them to recent Spitzer maps (Sect. 5). In Sect. 6 we take a look at the sub-mm properties of dense molecular clumps found in CO line observations, and in Sect. 7 we consider global properties of the complex, such as the total cloud mass, and perform radiative transfer modeling of the integrated spectral energy distribution to derive a global model for the CNC.

\section{Observations and data analysis}

The sub-mm observations of the CNC presented here were performed with the "Atacama Pathfinder EXperiment" (APEX), a 12-m radio telescope in Chile's Atacama desert, the best accessible site for submillimeter observations. The APEX telescope is described in detail in Güsten et al. (2006). We used the Large Apex BOlometer CAmera LABOCA (see Siringo et al. 2009), which operates in the atmospheric window at $870 \mu \mathrm{m}(345 \mathrm{GHz})$. It has 295 pixels arranged in a hexagonal layout consisting of a center channel and nine concentric hexagons. The angular resolution is 18.6", and the total field of view for LABOCA is $11.4^{\prime}$, making it an ideal instrument for mapping large regions with high sensitivity. At the $2.3 \mathrm{kpc}$ distance of the Carina Nebula, its angular resolution corresponds to a linear dimension of $0.2 \mathrm{pc}$. This is sufficient to resolve the structure of molecular clumps (i.e. relatively large dense clouds linked to the formation of small stellar clusters), but not the individual cloud cores (i.e. very compact clouds out of which individual stellar systems form), which have typical sizes of $\sim 0.1 \mathrm{pc}$ or less.

The LABOCA observations of the CNC discussed here were obtained on 22, 24 and 26 December 2007. For the mapping we employed the "raster map in spiral mode". The total area to be mapped was covered with a raster of pointings similar to, but larger than the one shown in Fig. 9 of Siringo et al. (2009). At each pointing, fully sampled maps of the total field-of-view of LABOCA were obtained by moving the telescope along a spiral pattern. The total on-source integration time used for our mapping of the $\mathrm{CNC}$ was $\approx 10 \mathrm{~h}$. The observing conditions were good, with a precipitable water vapor column of $<2 \mathrm{~mm}$.

The data were reduced with the BOlometer array Analysis software (BOA) package, following the procedures described in detail in Schuller et al. (2009). As the final product of the data analysis, a map with a pixel size of 6.07" (i.e. $\sim 3$ pixels per beam) was compiled. With a LABOCA beamsize of 392 square-arcseconds, the pixel-to-beam-size ratio for the transformation from surface brightness to integrated fluxes is 0.0941 beams/pixel.

The data were calibrated by applying an opacity correction, as determined from skydips observed typically every two hours (see Siringo et al. 2009). In addition, the flux calibration was regularly checked against primary calibrators (planets) or secondary calibrators (bright Galactic sources). The total calibration error should be lower than $15 \%$.

A fundamental limitation comes from the removal of correlated noise in the data reduction; because of this, our map is not sensitive to any structures with angular sizes larger than the array $\left(\geq 10^{\prime}\right)$ and can only partly recover emission on scales larger than $\approx 2.5^{\prime}$. As a consequence, possible uniform emission on angular scales $\gtrsim 2.5^{\prime}$ is filtered out and thus absent in our map. Therefore, the fluxes we measure in our map are always lower limits to the true sub-mm sky fluxes. The amount of this unseen flux depends on the (unknown) spatial distribution of the total emission in the map. If most of the emission comes from well localized, dense clouds, the missing flux will be very low or even negligible; the fluxes from individual compact $\left(<2.5^{\prime}\right)$ clouds can be reliably determined. If, on the other hand, there is bright large-scale emission, the measured flux can be considerably lower than the true total flux. These issues will be discussed in more detail in Sect. 7.1.

\section{General results}

The result of our LABOCA observations is a map of the $870 \mu \mathrm{m}$ emission, covering a total area of $1.25^{\circ} \times 1.25^{\circ}$; it is shown in Fig. 1. The rms noise level in the map is $\approx 20 \mathrm{mJy} / \mathrm{beam}$. For isolated compact clumps with assumed uniform temperatures of $T \approx 20-30 \mathrm{~K}$, this corresponds to a nominal sensitivity limit for the clump masses of $\sim 2 M_{\odot}$. This map provides the first spatially complete survey of the sub-mm emission in the CNC.

The maximum intensity in our map, $31.5 \mathrm{Jy} /$ beam, is found at the position of $\eta$ Car. Emission from clouds is seen with intensities up to $\approx 4 \mathrm{Jy} /$ beam. From these maximum cloud fluxes we 


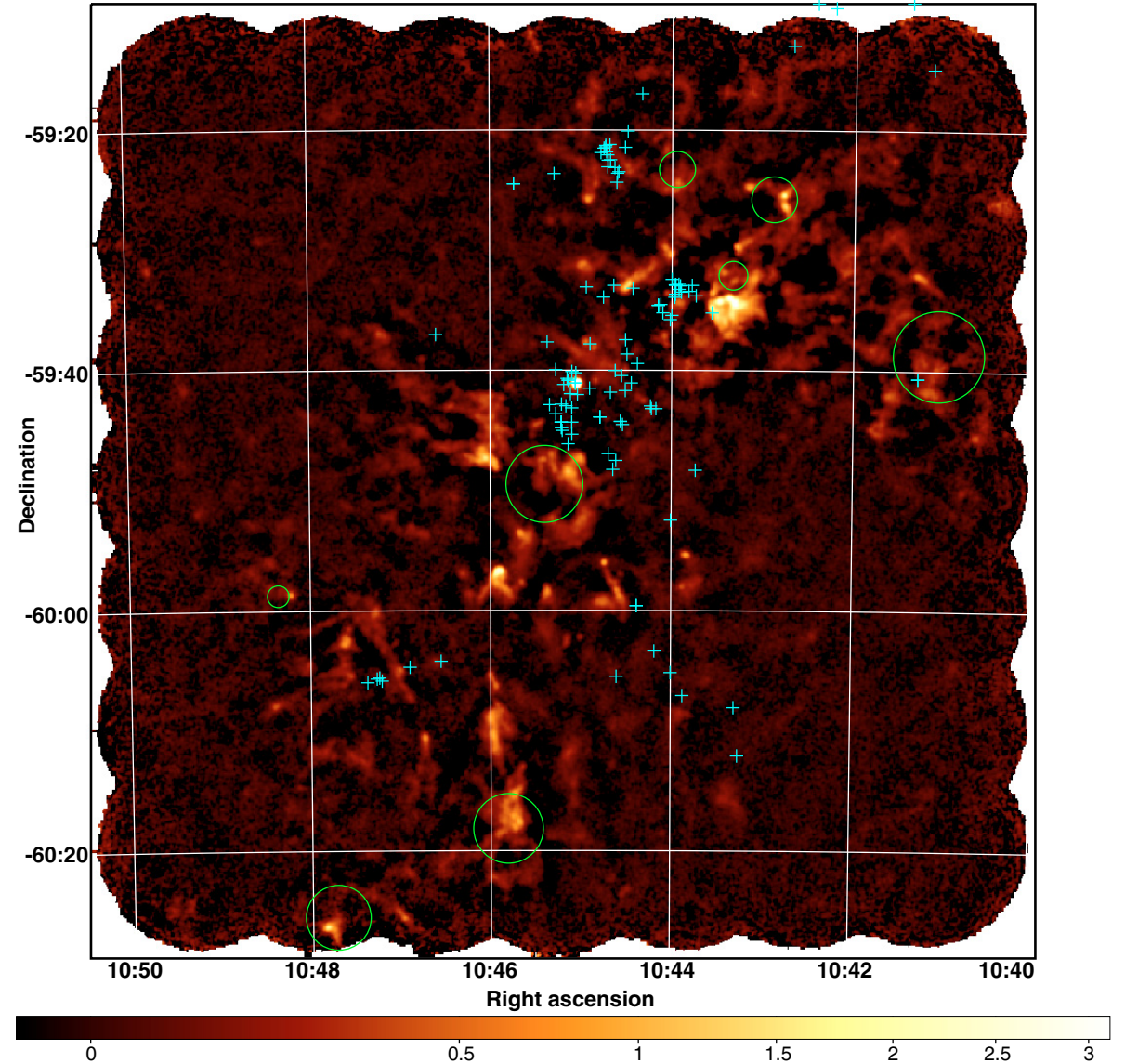

Fig. 1. False-color representation of the widefield LABOCA map of the Carina Nebula complex. The cyan crosses mark the positions of the massive (O- and early B-type) stellar members of the Carina Nebula from Smith (2006), the green circles mark the locations and radii of the dense $\mathrm{C}^{18} \mathrm{O}$ clumps detected by Yonekura et al. (2005) in this area. The field-of-view is $\approx 1.3^{\circ} \times$ $1.3^{\circ}$, corresponding to a size of $\approx 52 \times 52 \mathrm{pc}$ at the distance of the complex; the grid shows the J2000 coordinates. The units of the scale bar at the bottom are Jy/beam. can immediately infer (see, e.g., Eq. (3) in Schuller et al. 2009) that the optical depth of the emitting clouds in our map is small, $\tau_{870 \mu \mathrm{m}}<0.01$, i.e. all observed cloud emission is clearly in the optically thin limit.

In order to determine the total sub-mm flux in our LABOCA map, we excluded 25 pixel-wide edges of the mosaic, where the pixel values are often dominated by noise, and integrated all pixel values exceeding the $3 \sigma$ noise level of $60 \mathrm{mJy} / \mathrm{beam}$. We found a total $870 \mu \mathrm{m}$ flux of $1147 \mathrm{Jy}$ for our map.

\subsection{Nature of the observed sub-mm emission}

Two fundamentally different emission mechanisms can contribute to the observed $870 \mu \mathrm{m}$ continuum emission: thermal emission from dust, and free-free emission from ionized gas. The second mechanism is relevant in some parts of the CNC, where the numerous massive stars produce an extremely strong ionizing radiation field and create extended stellar wind bubbles. The $\mathrm{H} \alpha$ line is a good and easily detectable tracer of ionized gas. To localize $\mathrm{H} \alpha$ emission in the region we retrieved from the ESO archive images obtained with the Wide Field Imager at the MPI-2.2 $\mathrm{m}$ Telescope through an $\mathrm{H} \alpha$ filter $\left(\lambda_{\text {center }}=658.827 \mathrm{~nm}\right.$, $F W H M=7.431 \mathrm{~nm}$ ) for inspection. We also used the publicly available High Level Science Products of the HST ACS $\mathrm{H} \alpha$ Survey of the Carina Nebula ${ }^{1}$, Version 21 December 2009, created by Mutchler \& Bowers (2009). The diffuse emission

\footnotetext{
1 The data set and the processing are described at http://archive. stsci.edu/pub/hlsp/carina/hlsp_carina_hst_v2_readme. txt.
}

visible in the optical red DSS image (see Fig. 2) closely follows the $\mathrm{H} \alpha$ emission.

While these optical images trace the ionized gas, the distribution of the cold molecular gas can be inferred from molecular line maps. A comparison of the optical images with the $\mathrm{CO}$ maps presented in Yonekura et al. (2005) shows a fairly clear spatial anti-correlation between the ionized and the molecular gas in the CNC (see also Fig. 3 in Smith \& Brooks 2007, SB07 hereafter). Strong $\mathrm{H} \alpha$ emission is predominantly concentrated in the very center of the CNC, close to $\eta$ Car and the hot stars in $\operatorname{Tr} 16$, as well as near the massive cluster Tr 14. The CO emission, on the other hand, is concentrated in two large cloud structures, approximately to the north and south of $\eta$ Car.

Our comparison of the LABOCA map to the red optical image in Fig. 2 confirms this anti-correlation. Most of the sub-mm emitting clouds are located in regions more than a few arcminutes away from $\eta$ Car, where no (or at most very weak) $\mathrm{H} \alpha$ emission can be seen, and often correlate well with the molecular emission. In these regions, the sub-mm emission is dominated by thermal emission from cool dust. However, we also find some sub-mm emission in the central parts, very close to $\eta$ Car or $\operatorname{Tr} 14$. In these regions, the sub-mm emission is likely to be strongly contaminated and probably dominated by free-free emission, instead of thermal dust emission.

A quantitative estimate of the contributions of these different emission processes to the observed total sub-mm flux can be made in the following way: The total flux in the central $6^{\prime} \times 7^{\prime}$ area, which contains $\eta$ Car and the Keyhole Nebula and where most of the flux is likely free-free emission, is $70 \mathrm{Jy}$. The total flux in the whole map excluding this central area is $1077 \mathrm{Jy}$. 


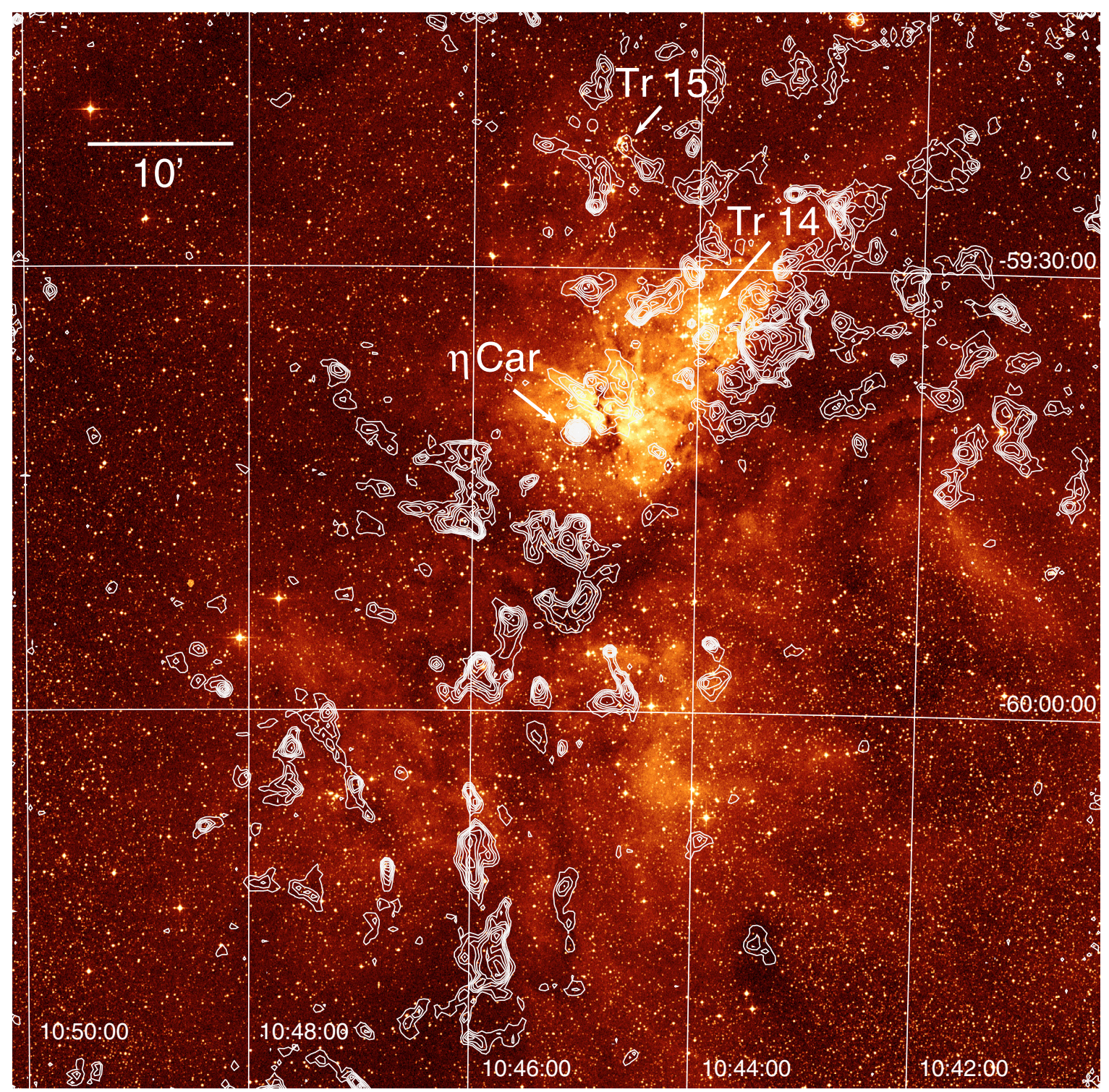

Fig. 2. False-color representation of the red optical Digitized Sky Survey image with contours of the LABOCA map overplotted. Here and (unless noted otherwise) in the following images, the first three contour levels are $0.06,0.12$, and $0.18 \mathrm{Jy} / \mathrm{beam}$, while the further levels increase by a factor of $\sqrt{2}$. The grid shows the $\mathrm{J} 2000$ coordinates.

Thus we conclude that about $94 \%$ of the observed sub-mm emission in our map comes from dusty clouds, whereas some $6 \%$ of the total flux is probably free-free emission.

\subsection{Fluxes and cloud masses}

The standard way to determine cloud masses from observed (sub-)mm fluxes for optically thin thermal dust emission is via a temperature-dependent scaling factor (see Hildebrand 1983), by using the formula

$M=\frac{D^{2} F_{v} R}{B_{v}\left(T_{\mathrm{d}}\right) \kappa_{v}}$,

where $D$ is the distance to the source (2300 pc in our case), $F_{v}$ is the observed spectral flux density, $R$ is the gas-to-dust mass ratio, $B_{v}\left(T_{\mathrm{d}}\right)$ is the blackbody spectral flux density for a dust temperature $T_{\mathrm{d}}$, and $\kappa_{v}$ is the dust emissivity. Following Schuller et al. (2009), we assume a gas-to-dust mass ratio of $R=100$ and a dust emissivity of $\kappa 870 \mu \mathrm{m}=1.85 \mathrm{~cm}^{2} \mathrm{~g}^{-1}$. The dust emissivity depends on the detailed properties of the dust grains; the value we use here is representative for relatively dense molecular clouds (Ossenkopf \& Henning 1994; Henning et al. 1995), but deviations by about a factor of 2 cannot be excluded.

The most important factor of uncertainty is the choice of the temperature. Firstly, the individual clouds in the CNC will have different temperatures, depending on (a) their location with respect to the luminous massive stars and on the cloud density, and (b) whether or not a cloud contains embedded protostars that heat it from inside. For some of the clouds in the CNC, temperature measurements are available: Brooks et al. (2003) found CO excitation temperatures of 20-30 K for the dense clouds near $\operatorname{Tr} 14$, and Yonekura et al. (2005) derived mean ${ }^{12} \mathrm{CO}$ excitation temperatures of $20-23 \mathrm{~K}$ for the large scale clouds and $\mathrm{C}^{18} \mathrm{O}$ excitation temperatures between $9 \mathrm{~K}$ and $28 \mathrm{~K}$ for dense clumps in the CNC. Owing to the quite strong and non-linear dependence of the mass estimates on the assumed cloud temperature ${ }^{2}$, we

${ }^{2}$ For example, a flux of $0.25 \mathrm{Jy}$ corresponds to a cloud mass of $23 M_{\odot}$ for $T=10 \mathrm{~K}, 7.3 M_{\odot}$ for $T=20 \mathrm{~K}$, and $4.2 M_{\odot}$ for $T=30 \mathrm{~K}$. 
have to consider the individual conditions in each cloud in order to derive meaningful mass estimates. Secondly, even for an individual compact cloud, the often used assumption of a spatially uniform "characteristic" temperature is probably not correct. A constant temperature may appear to be a reasonable approximation for dense clumps in nearby low-mass star-forming regions (where the external heating of the clouds is weak), but detailed modeling has shown that even in these relatively simple cases, slight deviations from isothermality by just a few degrees can easily lead to errors in the estimated clump masses by factors of $\sim 2$ (Stamatellos et al. 2007). In the harsh environment of the $\mathrm{CNC}$, where the level of cloud irradiation and heating by nearby hot and luminous stars is orders of magnitude higher than in quiescent regions, the assumption of a constant cloud temperature in the fairly large observed clumps can thus hardly be correct.

To summarize, the uncertainties about the dust opacities and the cloud temperatures will cause uncertainties of (at least) about a factor of $\sim 2-3$ for the mass estimates.

\subsection{Cloud column densities}

As discussed in detail in Sect. 3.1, the sub-mm flux we observe in most (but not all) parts of our map is most likely thermal dust emission. For a given cloud temperature, the observed $870 \mu \mathrm{m}$ intensities are then directly proportional to the column densities of the interstellar matter and thus the line-of-sight extinctions. These intensities can be converted to the beam-averaged hydrogen molecule column density via the formula

$$
N_{\mathrm{H}_{2}}=\frac{F_{v} R}{B_{v}\left(T_{\mathrm{d}}\right) \Omega \kappa_{v} \mu m_{\mathrm{H}}},
$$

where $\Omega$ is the beam solid angle and $\mu$ the mean molecular weight. Using again the parameters from Schuller et al. (2009), we find the following peak line-of-sight column densities for the two locations of maximum cloud emission: $N_{\mathrm{H}_{2}} \sim 6 \times 10^{22} \mathrm{~cm}^{-2}$ (corresponding to a visual extinction of $A_{V} \sim 65 \mathrm{mag}$ ) for the cloud near $\operatorname{Tr} 14$, and $N_{\mathrm{H}_{2}} \sim 4 \times 10^{22} \mathrm{~cm}^{-2}\left(A_{V} \sim 50 \mathrm{mag}\right)$ for the cloud at the Treasure Chest Cluster, when assuming cloud temperatures ${ }^{3}$ of $30 \mathrm{~K}$ in both cases.

A very prominent feature in optical images of the $\mathrm{CNC}$ is the $V$-shaped dark cloud with its tip a few arcminutes south of $\eta$ Car. The LABOCA map shows that this feature consists of a number of different clouds. Typical intensities in the diffuse parts of these clouds are in the range $0.2-0.5 \mathrm{Jy} / \mathrm{beam}$, which (assuming again a typical dust temperature of $30 \mathrm{~K}$ ) correspond to column densities of $N_{\mathrm{H}_{2}} \sim 3-7 \times 10^{21} \mathrm{~cm}^{-2}$ and extinctions of $A_{V} \sim 3-7 \mathrm{mag}$. These fairly moderate values agree well with the fact that the $V$-shaped dark cloud is transparent and thus invisible in near-infrared images of the CNC.

\section{Results for individual parts of the complex}

\section{1. $\eta$ Car}

The famous object $\eta$ Car is a close binary with strong wind-wind interaction (see, e.g., Groh et al. 2010). The (sub-)mm emission is known to be free-free emission from ionized gas in its stellar wind.

In our LABOCA map, we measure a source flux of $\approx 43 \mathrm{Jy}$ in a circular aperture of radius $26^{\prime \prime}$ for $\eta$ Car. This flux value seen on 26 December 2007 is nearly identical to the flux derived by

\footnotetext{
3 The choice of $T=30 \mathrm{~K}$ was made because these clouds are irradiated (=heated) by stars either near or within these clouds.
}

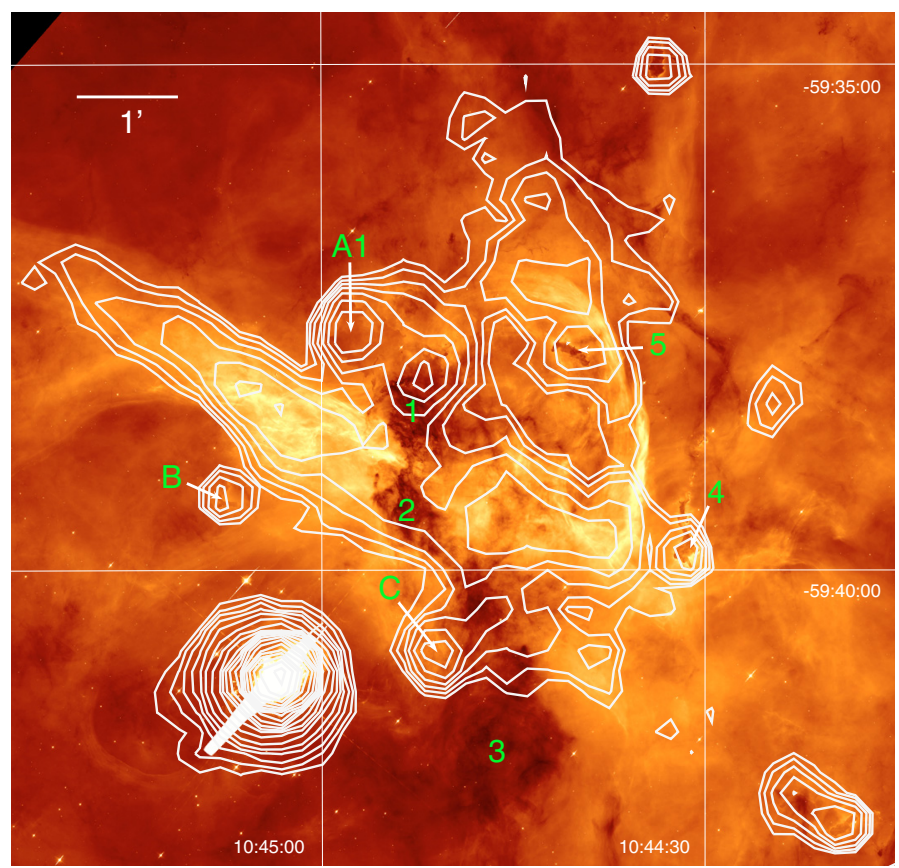

Fig. 3. False-color representation of the HST $\mathrm{H} \alpha$ image of $\eta$ Car and the Keyhole Nebula with superposed contours of the LABOCA map. The numbers 1-5, A1, B, and C refer to the CO clumps in the Keyhole Nebula defined in Cox \& Bronfman (1995). The contour levels in this image start at $0.06 \mathrm{Jy} /$ beam and increase on a logarithmic scale by factors of 1.364 (i.e. $0.135 \mathrm{dex}$ ).

Gomez et al. (2010) (42 Jy in a 26" radius aperture) from another LABOCA observation obtained on 23 July 2007. This observed constancy may be interesting in the context of the known strong temporal variability of the (sub-)mm fluxes by at least a factor of 2 (see, e.g., discussion in Brooks et al. 2005) and also with respect to the detection of a recent significant decrease of the strength of major stellar-wind emission lines in the spectrum of $\eta$ Car, which seem to suggest a recent and very rapid decrease in the wind density (Mehner et al. 2010).

\subsection{Clouds near $\operatorname{Tr} 16$ and the Keyhole Nebula region}

The loose cluster Tr 16 is located in the center of the Carina Nebula and includes the optically dominant star $\eta$ Car as well as the majority of the O-type stars in the complex.

The Keyhole Nebula Region, immediately to the north of $\eta \mathrm{Car}$, is the best-observed part of the $\mathrm{CNC}$ and the only region that has already been studied in detail at sub-mm and mm wavelengths. The comparison of our LABOCA map to the optical $\mathrm{H} \alpha$ mosaic obtained with the HST is shown in Fig. 3. Comparison of our LABOCA map to the SIMBA $1.2 \mathrm{~mm}$ map of Brooks et al. (2005) generally shows a good agreement in observed cloud morphologies. This central region was recently studied with independent LABOCA observations by Gomez et al. (2010). With a noise level of $\approx 40 \mathrm{mJy} /$ beam, their data are about half as sensitive as ours. A comparison of our map to their results shows good agreement in the general morphology and levels of the submm emission, although our map reveals (as expected) weaker emission structures. Gomez et al. (2010) discuss in detail the relation between the sub-mm emission and the emission from ionized gas, as seen in $\mathrm{H} \alpha$ in the central few arcminutes around $\eta$ Car. For most of the cloud structures they found a close correlation between ionized gas, emission at both $\mathrm{mm}$ and radio 
Table 1. LABOCA fluxes of the CO clumps in the Keyhole Nebula listed in Cox \& Bronfman (1995).

\begin{tabular}{lrrrr}
\hline \hline Clump & $M(\mathrm{CO})$ & $F(870 \mu \mathrm{m})$ & $M_{870}(20 \mathrm{~K})$ & $M_{870}(30 \mathrm{~K})$ \\
$\#$ & {$\left[M_{\odot}\right]$} & {$[\mathrm{Jy}]$} & {$\left[M_{\odot}\right]$} & {$\left[M_{\odot}\right]$} \\
\hline CB-A1 & 14 & $0.54 \pm 0.08$ & 15.8 & 9.1 \\
CB-B & 3 & $0.20 \pm 0.03$ & 5.8 & 3.4 \\
CB-C & 6 & $0.25 \pm 0.04$ & 7.3 & 4.2 \\
CB-1 & 17 & & & \\
CB-2 & 11 & & & \\
CB-3 & 4 & $<0.06$ & $<1.8$ & $<1.0$ \\
CB-4 & 6 & $0.29 \pm 0.04$ & 8.5 & 4.9 \\
CB-5 & 1 & $0.13 \pm 0.02$ & 3.8 & 2.2 \\
\hline
\end{tabular}

wavelengths, and the sub-mm emission. Together with the clear lack of strong molecular line emission in this region (Yonekura et al. 2005) this suggests that the $870 \mu \mathrm{m}$ emission here originates predominantly from ionized material, and not from dust.

However, some weak molecular line emission is nevertheless present in this region: Cox \& Bronfman (1995) discovered $\mathrm{CO}$ emission at eight different positions near the Keyhole Nebula, including three peaks aligned with the centers of the optically darkest parts of the Keyhole Nebula (their positions 1, 2 , and 3). In the following discussion of the sub-mm emission seen in our LABOCA map at the location of these clumps, we use their clump numbers with the prefix "CB". We clearly see compact $870 \mu \mathrm{m}$ emission at the locations of all their clumps with the exception of CB-1, CB-2, and CB-3. In order to estimate the fluxes of the sub-mm detected $\mathrm{CO}$ clumps, which appear as compact sources superposed on top of fairly strong and inhomogeneous diffuse emission, we performed aperture photometry at these positions, using circular apertures with radii of 2.4 pixels and annular sky regions between 1.5 times and twice the aperture radius. Note that the inhomogeneous diffuse background emission in this area is a serious complication for flux determinations, and thus the expected uncertainties of our background-subtracted aperture fluxes listed in Table 1 are probably not less than $\sim 30 \%$. We also list clump masses derived via Eq. (1), assuming two different values for the temperature, $20 \mathrm{~K}$ and $30 \mathrm{~K}$. The mass estimates based on $T=20 \mathrm{~K}$ are always higher than the masses derived from the $\mathrm{CO}$ lines by Cox \& Bronfman (1995), but the estimates based on $T=30 \mathrm{~K}$ agree within a factor of $\sim 2$ to the $\mathrm{CO}$ masses. This suggests that the material in these clumps is fairly warm, probably because of the close proximity to the very luminous stars in this area, which strongly heat them.

For the three CO clumps coinciding with the optically dark Keyhole nebula features, CB-1, CB-2, and CB-3, no clear detection of sub-mm emission can be established from our data. In deed, we find local minima of the extended sub-mm emission in the Keyhole area at these three positions. The strong $870 \mu \mathrm{m}$ emission slightly to the north of CB-1 is related to the clump CB-A2. More quantitative statements are hard to make for CB-1 and $\mathrm{CB}-2$, because these regions are strongly affected by blending with the emission from the long linear structure, Car II, to the north of $\eta$ Car. CB-3, on the other hand, is located at a less confused region of our map and can be characterized in more detail. We find no significant sub-mm emission at this position; with a maximum intensity of $0.04 \mathrm{Jy} / \mathrm{beam}$, all pixel values in this region remain well below the $3 \sigma$ noise limit of $0.06 \mathrm{Jy} /$ beam. Assuming an upper limit for the sub-mm flux of CB-3 of $0.06 \mathrm{Jy}$, we can confirm that given a mass of $4 M_{\odot}$ as derived by

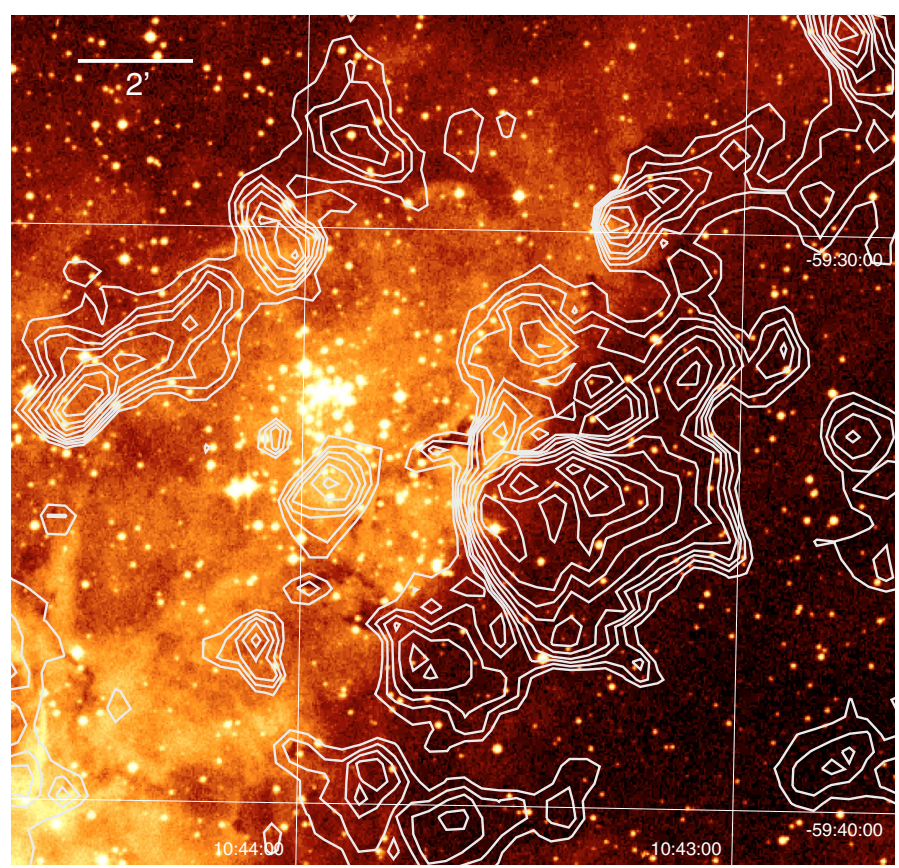

Fig. 4. False-color representation of the red DSS image for the region near $\operatorname{Tr} 14$ with superposed contours of the LABOCA map.

Cox \& Bronfman (1995), the temperature of this clump must be $\leq 12 \mathrm{~K}$ to explain the non-detection in our sub-mm map.

This cold temperature suggests that this, and the other two non-detected dark clouds are only seen this close to the massive stars in projection, but are actually located at least a few parsec in front of $\operatorname{Tr} 16$; otherwise, one would expect considerably higher cloud temperatures because of the strong radiative heating.

To conclude the discussion of this central region, we note that all clouds in this area are only of rather low mass. The original mass out of which the numerous massive stars formed has been already nearly completely dispersed, leaving only a few small globules behind.

\subsection{Clouds near $\operatorname{Tr} 14$}

The cluster $\operatorname{Tr} 14$ is the second most massive cluster in the Carina Nebula; with 10 O-type stars it is about a factor of four less massive than $\operatorname{Tr} 16$, but its spatial configuration is considerably more compact. The brightest sub-mm emitting cloud complex in our map is found a few arcmin to the west of Tr 14. Together with a number of more diffuse clouds to the southeast, this complex constitutes the western part of the optically prominent $V$-shaped dark nebula in the CNC.

Although the massive cluster $\operatorname{Tr} 14$ contains a substantial number of hot O-stars that produce large amounts of ionizing flux, the situation here seems to be quite different from that in the Keyhole Nebula region. The comparison of the LABOCA maps and the optical image (Fig. 4) clearly shows a strong anticorrelation of $\mathrm{H} \alpha$ and sub-mm emission. This may be at least partly explained by the fact that the cloud to the west of $\operatorname{Tr} 14$ is much denser and more massive than the clouds in the Keyhole area, and the ionizing radiation therefore can affect only the cloud surface, but not penetrate the cloud. The edge where this cloud faces the stellar clusters is the site of a prominent Photon Dominated Region (PDR), which has been studied in some detail by Brooks et al. (2003) and Kramer et al. (2008). From 
molecular line observations of the dense cloud to the west of $\operatorname{Tr} 14$, Brooks et al. (2003) estimated a total cloud mass of $\sim 20000 M_{\odot}$ and determined $\mathrm{CO}$ excitation temperatures of $20-30 \mathrm{~K}$.

Integrating in our LABOCA map over all pixel values above the $3 \sigma$ noise level with a radius of $4^{\prime}$ from the peak of emission, we find a $870 \mu \mathrm{m}$ flux of $157 \mathrm{Jy}$. Most of the flux (98 Jy) comes from the brightest central parts of the emission at levels above $0.5 \mathrm{Jy} /$ beam. Assuming a cloud temperature of $20 \mathrm{~K}$, the total cloud flux of $157 \mathrm{Jy}$ corresponds to a cloud mass of $\sim 4600 M_{\odot}$, i.e. a value substantially lower than the estimate based on the molecular line observations. Considering our above estimate for the column density through the densest parts of this cloud, $N_{\mathrm{H}_{2}} \sim$ $6 \times 10^{22} \mathrm{~cm}^{-2}$, it may well be that the temperature in the inner, dense parts of the cloud, where most of the mass resides, is lower than $20 \mathrm{~K}$. Assuming a characteristic dust temperature of $10 \mathrm{~K}$ yields a mass of $\sim 14400 M_{\odot}$. Considering the uncertainties in the mass determinations described above, this value would agree reasonably well with the mass estimate from $\mathrm{CO}$.

Finally, we consider the relation between the stellar cluster $\operatorname{Tr} 14$ and the surrounding clouds. The area within $\lesssim 2^{\prime}$ of the cluster center is largely devoid of sub-mm emission, suggesting that the original cloud has been largely dispersed by the feedback from the massive stars. In a recent study of the Tr 14 stellar population, Sana et al. (2010) claimed an age of no more than 0.3-0.5 Myr for the cluster. If this very young age is true, one would expect to see the expelled remnants of the original cloud out of which the cluster formed to be still relatively close to the cluster. Might the elongated cloud complexes to the south-west and north-east of $\operatorname{Tr} 14$ be this expelled material? We can investigate this question by comparing the masses of these clouds to the expected mass of expelled gas in the formation of a dense cluster. The total stellar mass of $\operatorname{Tr} 14$ is $M_{\text {stars }} \sim 4000 M_{\odot}$ (Sana et al. 2010). Assuming that $\$ 30 \%$ of the original cloud mass have been transformed into stars (i.e. a star formation efficiency of $\$ 30 \%$, see Lada \& Lada 2003), and the other $\sim 70 \%$ have been expelled, the mass of the expelled clouds should be $\gtrsim 9300 M_{\odot}$.

This estimate is actually consistent with the total mass of the two largest cloud complexes seen near Tr 14 in Fig. 4: the total $870 \mu \mathrm{m}$ flux of the elongated cloud northeast of $\operatorname{Tr} 14$ is $\approx 35 \mathrm{Jy}$, corresponding to a cloud mass of $\sim 1000 M_{\odot}$ (for an assumed temperature of $20 \mathrm{~K}$ ). The total mass of the cloud complex to the southwest of $\operatorname{Tr} 14$ is, as estimated above, $\sim 14000 M_{\odot}$. We thus conclude that the total mass of surrounding clouds within a few parsecs from $\operatorname{Tr} 14$ is about as large as expected, if we assume these clouds to be the remnant of the cloud out of which Tr 14 formed and have been recently expelled by the action of the massive stars in $\operatorname{Tr} 14$.

The spatial distribution of these clouds, however, does not follow the morphology expected for a (more or less) homogeneous bubble around the cluster, as seen in many other massive star forming regions (e.g., Deharveng et al. 2009). Inspection of Fig. 4 shows no circular alignment of clouds around $\operatorname{Tr} 14$, but rather a morphology suggesting a "broken ring", which is open in the northwestern and southeastern direction. A possible explanation of this morphology is that the clouds form a thick ring-like structure around $\operatorname{Tr} 14$, which we see nearly edgeon. Beaumont \& Williams (2010) recently suggested that many apparent bubbles around young clusters are actually not threedimensional spheres, but instead more or less two-dimensional thick rings. They claim that this could be the result of star formation and stellar feedback within a flattened, sheet-like molecular cloud. The spatial configuration of the clouds around Tr 14 seem to fit to this model.

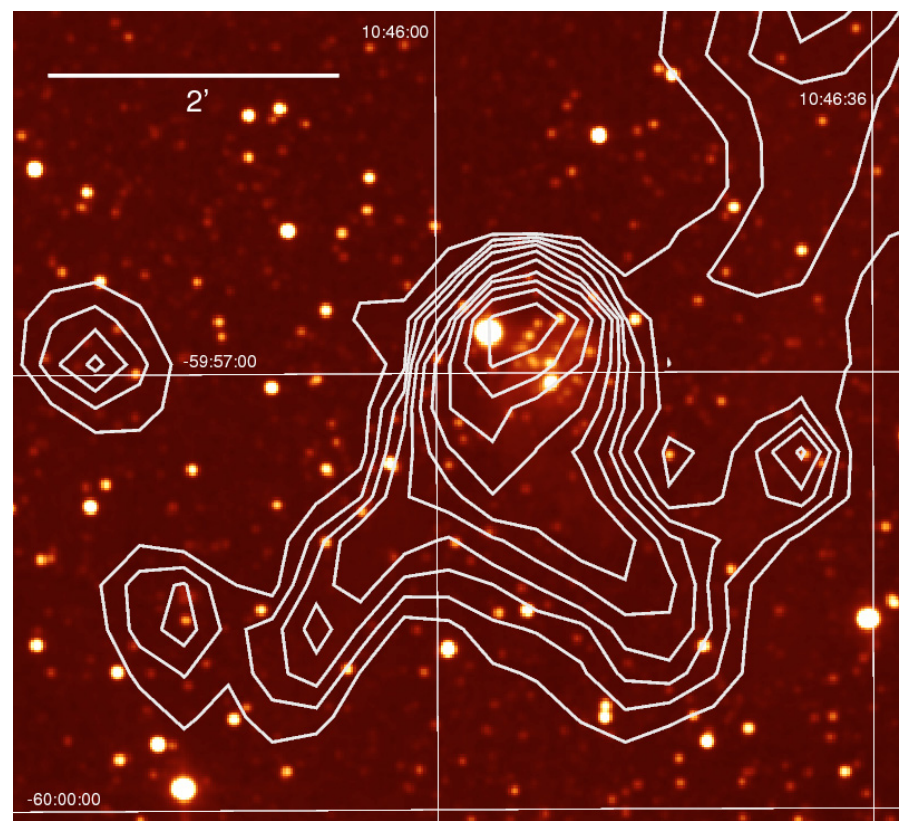

Fig. 5. False-color representation of the 2MASS $K$-band image of the area around the Treasure Chest Cluster (located at RA (J2000) = $10^{\mathrm{h}} 45^{\mathrm{m}} 54^{\mathrm{s}}$, Dec $\left.=-59^{\circ} 56^{\prime} 50^{\prime \prime}\right)$ with superposed contours of the LABOCA map.

\subsection{Clouds near the cluster $\operatorname{Tr} 15$}

The northern cluster $\operatorname{Tr} 15$ is considerably smaller and less prominent than $\operatorname{Tr} 14$ and $\operatorname{Tr} 16$. Containing 6 O-type stars, its total ionizing radiation output is about one order of magnitude weaker than for $\operatorname{Tr} 14$ and $\operatorname{Tr} 16$. The cluster is thought to be somewhat older than $\operatorname{Tr} 14$ and $\operatorname{Tr} 16$, probably around 6-8 Myr (see, e.g. Tapia et al. 2003).

Only moderately bright sub-mm emission is seen in this area (see Fig. 2). None of the clouds near Tr 15 shows morphological indications of feedback from stars in Tr 15. The cloud to the south-east of the cluster shows a prominent pillar-like structure, but it points toward the south, clearly suggesting that it is irradiated by massive stars in the south (presumably in $\operatorname{Tr} 16$ ), not from $\operatorname{Tr} 15$.

One elongated dusty cloud, pointing toward the southwestern direction, covers the western half of the cluster area. It is interesting to note that many of the OB stars in $\operatorname{Tr} 15$ are apparently aligned along the northeastern rim of this cloud. This may be an extinction effect, suggesting perhaps that the cloud is only seen in projection in front of $\operatorname{Tr} 15$ and is not physically associated.

\subsection{Clouds in the South Pillars}

The so-called South Pillars are a complex of strongly irradiated clouds south of $\eta$ Car, featuring very prominently in Spitzer images. Our LABOCA map reveals several large and dense, as well as numerous small and more diffuse clouds in this area. A number of these clouds show prominent cometary shapes pointing towards $\eta$ Car. In many cases, the sub-mm morphology closely follows the shape of the cloud surfaces as seen in the Spitzer images; this will be discussed in more detail in Sect. 5. The two northernmost larger clouds in this region constitute the eastern part of the $V$-shaped dark feature seen in the optical images.

A particularly interesting part of the South Pillars is the Treasure Chest, a dense cluster of some 200 stars embedded in 
a compact nebula at the head of a large dust pillar (Smith et al. 2005). The brightest object in this cluster is the O9.5 V star CPD $-59^{\circ} 2661$, that ionizes a small HII region inside a $\sim 40^{\prime \prime}$ diameter cavity near the western edge of the dust pillar, in which the cluster is located. The edges of this cavity can be well seen in the narrowband images presented in Smith et al. (2005, their Fig. 3). The cluster seems to be extremely young $(\$ 0.1 \mathrm{Myr})$ and the young stars show strong reddening with extinction values up to $A_{V} \sim 50 \mathrm{mag}$.

Our LABOCA data reveal the detailed morphology of the associated dust cloud (see Fig. 5) out of which this cluster recently formed. The large scale structure of the $870 \mu \mathrm{m}$ emission shows a cometary shape, with peak intensity just to the north and some $15^{\prime \prime}$ to the east of the infrared cluster. At the position of the cavity surrounding the embedded cluster, the sub-mm emission shows a "hole" and is $\sim 3 \times$ weaker than on the eastern side. This suggests that the cavity takes up a large fraction of the full depth of the dust cloud, and is not just a minor disturbance at the surface of the cloud.

Our column density estimate for the brightest part of the cloud, $N_{\mathrm{H}_{2}} \sim 4 \times 10^{22} \mathrm{~cm}^{-2}$, corresponds to visual extinction values of $A_{V} \sim 50 \mathrm{mag}$ and agrees very well with the maximum extinction found by Smith et al. (2005) for the stars in this region. Proceeding to the south, the structure of the cloud shows a bifurcation; the western arm is denser and thicker than the eastern arm.

To derive an estimate for the total mass of the dust cloud, we integrated the $870 \mu \mathrm{m}$ emission above the $3 \sigma$ noise level over $\mathrm{a} \approx 2^{\prime} \times 3.5^{\prime}$ box including the brightest part of the emission, finding a flux of $30.2 \mathrm{Jy}$. Assuming a cloud temperature of $20 \mathrm{~K}$, this suggests a cloud mass of $\sim 880 M_{\odot}$.

\section{Cloud morphology from the combination of the Spitzer and the LABOCA images}

The combination of our LABOCA data with the existing Spitzer images provides a very good way to study the morphology of the clouds. The $8 \mu \mathrm{m}$ emission in the IRAC 4 band is dominated by polyaromatic hydrocarbon emission, which is excited by the UV irradiation, and thus reveals the detailed structure of the cloud surfaces. The $870 \mu \mathrm{m}$ emission, on the other hand, traces the cold dust inside the dense central cloud regions. The full extent of the Carina Nebula has been mapped with IRAC, and we retrieved these data ${ }^{4}$ from the Spitzer data archive for analysis. Below, we discuss interesting features resulting from this investigation.

In Fig. 6 we compare the LABOCA data to the Spitzer $8.0 \mu \mathrm{m}$ image of the South Pillars region. In many parts of this region we find a remarkable resemblance between the morphology in the Spitzer and the LABOCA images. Essentially all clouds that emit brightly in the Spitzer image do also clearly show $870 \mu \mathrm{m}$ emission.

On the other hand, not all of the dense dusty clouds revealed by LABOCA are visible in the Spitzer images. An especially notable example is the cloud marked with "IRDC" in Fig. 6, near the western edge of the South Pillars. A more detailed view and comparison to images in other wavelengths are provided in Fig. 7. In the LABOCA map, this cloud shows a double-peaked structure. In the Spitzer $8 \mu \mathrm{m}$ IRAC map as well as the $24 \mu \mathrm{m}$ MIPS map, these two $870 \mu \mathrm{m}$ peaks correspond to very dark shadows, which are located at

\footnotetext{
4 These IRAC data were obtained in July 2008 in the program "Galactic Structure and Star Formation in Vela-Carina" (PI: Steven Majewski, Prog-ID: 40791).
}

$\mathrm{RA}=10^{\mathrm{h}} 43^{\mathrm{m}} 18.2^{\mathrm{s}}$, Dec $=-60^{\circ} 15^{\prime} 59^{\prime \prime}$, and RA $=10^{\mathrm{h}} 43^{\mathrm{m}} 24.9^{\mathrm{s}}$, Dec $=-60^{\circ} 15^{\prime} 40^{\prime \prime}$. The western [eastern] peak shows a flux maximum of 0.25 [0.19] Jy/beam, which translates into a column density of $N_{\mathrm{H}_{2}} \approx 2[1.5] \times 10^{22} \mathrm{~cm}^{-2}$, corresponding to a visual extinction of $A_{V} \approx 22$ [17] mag.

The optical DSS image shows that this cloud corresponds to a very prominent dark globule in the southern part of the CNC. This cloud seems to be a good example of an infrared dark cloud, i.e. particularly dense and cold clouds, which are thought to be the birth places of massive stars (e.g., Carey et al. 1998; Rathborne et al. 2006; Rygl et al. 2010). The integrated $870 \mu \mathrm{m}$ flux of this cloud is $3.5 \mathrm{Jy}$, which corresponds to a cloud mass of $\sim 320 M_{\odot}$ for $T=10 \mathrm{~K}$.

Finally, in Fig. 8 we consider the small scale structure of the massive cloud complex to the west of $\operatorname{Tr} 14$. The eastern edge, where the cloud is strongly irradiated by the massive stars in $\operatorname{Tr} 14$ and where a bright PDR can bee seen in the optical images, the rim of the cloud is very sharp. The interior of the cloud complex seems to consist of at least five bright clumps. To the north and the southeast of this dense cloud complex, weaker diffuse emission is visible. This may be material that was evaporated at the eastern edge of the cloud in the PDR and is now streaming away in the directions perpendicular to the impacting radiation field from $\operatorname{Tr} 14$.

\section{Sub-mm emission from $\mathrm{CO}$ molecular cloud clumps}

Yonekura et al. (2005) detected 15 individual cloud clumps ${ }^{5}$ in their $\mathrm{C}^{18} \mathrm{O}$ map ${ }^{6}$; eight of these (number 8-15) are located in the field-of-view of our LABOCA map and are marked by the red circles in Fig. 1. Sub-mm emission is clearly detected at the locations of all eight clumps. In five cases (clumps 9, 11, 13, 14, and 15) our LABOCA map shows strong and compact sub-mm emission, as expected for high-density clumps. At the location of clumps 8,10 , and 12 , however, we see only diffuse sub-mm emission.

In order to determine the sub-mm fluxes of the clumps, we used the positions and sizes (the beam-deconvolved radii) of the clumps reported in Table 3 of Yonekura and integrated all pixels values above the $3 \sigma$ noise level. For the clumps with clear peaks in the sub-mm map we centered the integration regions on these peaks. The resulting fluxes are given in Table 2. We also list the corresponding masses computed from these fluxes, assuming two different temperature values, $T=20 \mathrm{~K}$ and $T=10 \mathrm{~K}$.

Comparison to the masses determined by Yonekura from their $\mathrm{C}^{18} \mathrm{O}$ map shows that the sub-mm mass estimates for $T=$ $20 \mathrm{~K}$ are always substantially lower than the $\mathrm{C}^{18} \mathrm{O}$ masses. With $T=10 \mathrm{~K}$ we find a much better agreement; the mass estimates from the sub-mm data and the $\mathrm{C}^{18} \mathrm{O}$ data generally agree within a factor of $\sim 2$. Although this seems to be a relatively good match, we note that the assumed very cold $T=10 \mathrm{~K}$ temperatures appear to be in conflict with the $\mathrm{CO}$ excitation temperatures, which are $\geq 20 \mathrm{~K}$ for all but one of these clumps. This apparent inconsistency may perhaps indicate that the derived $\mathrm{CO}$ excitation temperatures do not well represent the characteristic temperature at which most of the cold dust mass resides.

\footnotetext{
5 We note that Yonekura et al. (2005) denoted these structures as "cores"; however, with a size scale of $\sim 2$ pc, these clouds are not cores (which have typical sizes of $\lesssim 0.2 \mathrm{pc}$ ) but rather clumps.

${ }^{6}$ With a half-power beam width of $2.7^{\prime}$ the spatial resolution of the $\mathrm{C}^{18} \mathrm{O}$ map is about nine times lower than that of our LABOCA map.
} 


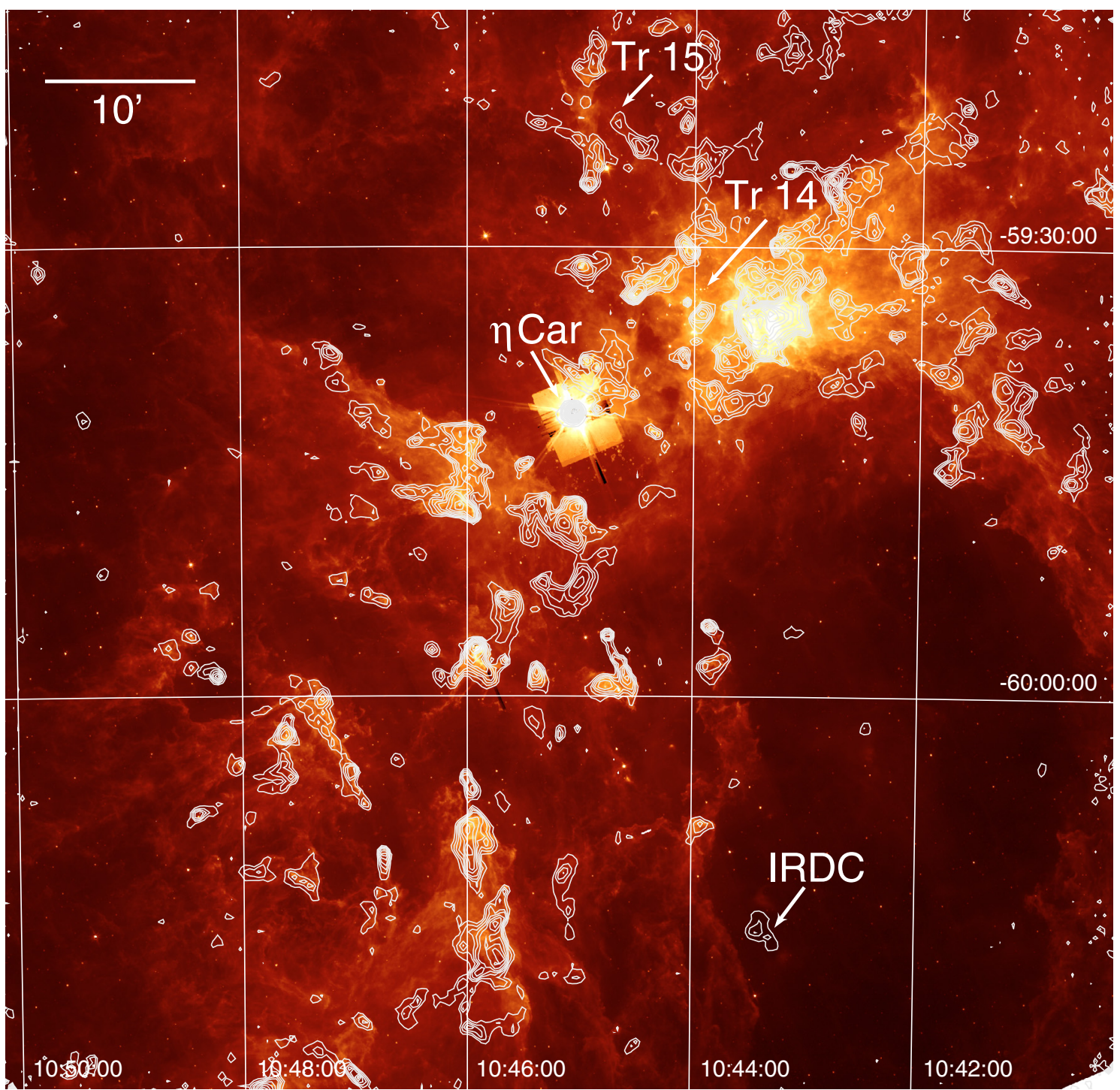

Fig. 6. False-color representation of the $8.0 \mu \mathrm{m}$ Spitzer image with superposed contours of the LABOCA map. In the area within a few arcminutes from $\eta$ Car the very bright infrared emission caused strong artifacts in the Spitzer image. A grid of J2000 coordinates is shown.

\section{Global properties of the Carina Nebula complex}

Our wide-field LABOCA map yields for the first time a direct measurement of the total sub-mm emission from the CNC. It represents a missing piece of the puzzle for the investigations of the global properties of the complex, in particular for the total luminosity and its spectral energy distribution, and for the total mass and its distribution in the different phases, i.e. dust, molecular, and atomic gas.

\subsection{The total dust and gas mass}

We can use our sub-mm map to derive an estimate of the total mass of dust and gas in the Carina Nebula. We note again that our LABOCA map is not sensitive to possibly existing widespread and spatially homogeneous sub-mm emission on large angular scales, $>2.5^{\prime}$; our measured sub-mm fluxes are therefore only lower limits to the true flux. In principle, if most of the cloud mass is concentrated into dense compact structures, the fraction of unrecovered large-scale flux may be very small (see, e.g., Maruta et al. 2010). However, the CNC clearly shows wide-spread far-infrared emission (as visible, e.g., in the
IRAS images) on scales of tens of arcminutes, and therefore we expect that the missing flux is not insignificant. On the other hand, it appears likely that some part of this large scale emission arises from distant clouds in the background; owing to its position very close to the Galactic plane $\left(l \approx-0.6^{\circ}\right)$ and near the tangent point of the Sagittarius-Carina spiral arm, the Galactic background emission at the location of the Carina Nebula must be fairly high.

As determined in Sect. 3, the total flux in our map originating from dusty clouds (and not from free-free emission) is $1077 \mathrm{Jy}$. To illustrate how sensitively the corresponding mass estimate depends on the assumed dust temperature, we consider three different temperature values, and find that the measured flux corresponds to (dust + gas) masses of $99000 M_{\odot}, 31400 M_{\odot}$, or $18100 M_{\odot}$ for 10,20 , or $30 \mathrm{~K}$, respectively. As our best estimate we assume here a total mass of the cold dusty clouds traced by LABOCA of $\sim 60000 M_{\odot}$.

The total mass of molecular gas in the CNC has been derived from the $\mathrm{CO}$ observations by Yonekura et al. (2005). Since their full map size is considerably larger than the field-of-view of our LABOCA map, we added their CO masses for their subregions $1,2,3$, and 7 , which cover approximately the field of our 

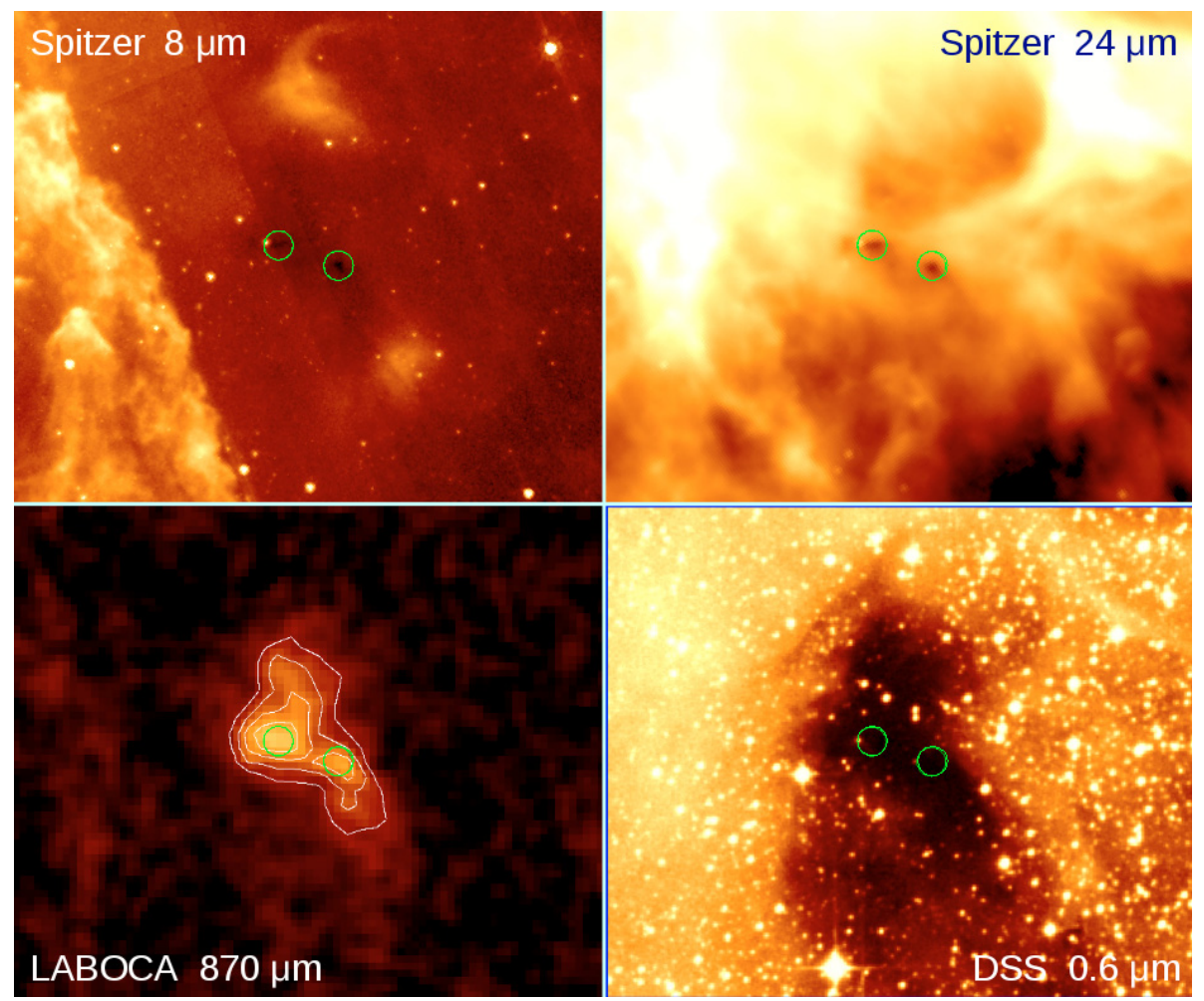

Fig. 7. False-color images of the region around the infrared dark cloud, seen in the Spitzer $8 \mu \mathrm{m}$ IRAC map (upper left), the $24 \mu \mathrm{m}$ MIPS map (upper right), the LABOCA map (lower left), and the optical DSS image (lower right). In each image, the field-of-view is $\approx 8.2^{\prime} \times 6.6^{\prime}$, north is up, east to the left. The contour levels superposed onto the LABOCA map are 0.06, $0.09,0.12$, and $0.15 \mathrm{Jy} /$ beam. The two peaks are marked by green circles.

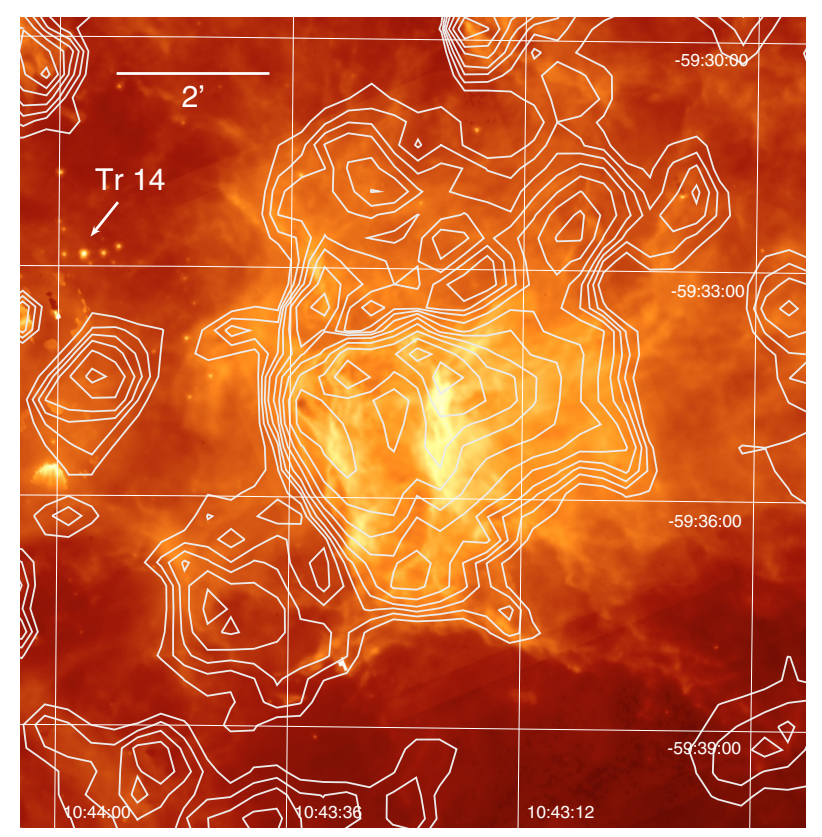

Fig. 8. False-color representation of the $8.0 \mu \mathrm{m}$ Spitzer image of the cloud west of $\operatorname{Tr} 14$ with superposed contours of the LABOCA map.

LABOCA map. This yields a total mass of $141000 M_{\odot}$ based on their ${ }^{12} \mathrm{CO}$ map, $63000 M_{\odot}$ from their ${ }^{13} \mathrm{CO}$ map, and $22000 M_{\odot}$ from their $\mathrm{C}^{18} \mathrm{O}$ map. These numbers suggest that a large fraction of the cloud mass resides in clouds of moderate density (as traced by ${ }^{12} \mathrm{CO}$ ), whereas the denser gas (as traced by ${ }^{13} \mathrm{CO}$ ) and the very dense clouds traced by $\mathrm{C}^{18} \mathrm{O}$ contain progressively smaller fractions of the total mass. Interestingly, our mass estimate for the cloud emission seen by LABOCA of $\sim 60000 M_{\odot}$ agrees fairly well to the mass in dense, well localized clouds as traced by ${ }^{13} \mathrm{CO}$. Comparison to the ${ }^{12} \mathrm{CO}$ mass suggests that our
Table 2. LABOCA fluxes and mass estimates of the $\mathrm{C}^{18} \mathrm{O}$ clumps detected by Yonekura et al. (2005).

\begin{tabular}{rrrrrr}
\hline \hline Clump & $T_{\text {ex }}$ & $M\left(\mathrm{C}^{18} \mathrm{O}\right)$ & $F(870 \mu \mathrm{m})$ & $M_{870}(20 \mathrm{~K})$ & $M_{870}(10 \mathrm{~K})$ \\
$\#$ & {$[\mathrm{~K}]$} & {$\left[M_{\odot}\right]$} & {$[\mathrm{Jy}]$} & {$\left[M_{\odot}\right]$} & {$\left[M_{\odot}\right]$} \\
\hline 8 & 24 & 3700 & $28.4 \pm 4.3$ & 829 & 2612 \\
9 & 21 & 1400 & $20.3 \pm 3.0$ & 593 & 1868 \\
10 & 25 & 520 & $11.9 \pm 1.8$ & 347 & 1093 \\
11 & 22 & 1400 & $10.7 \pm 1.6$ & 312 & 983 \\
12 & 20 & 2600 & $37.5 \pm 5.6$ & 1095 & 3450 \\
13 & 20 & 4200 & $41.5 \pm 6.2$ & 1212 & 3819 \\
14 & 9 & 450 & $2.9 \pm 0.4$ & 85 & 267 \\
15 & 19 & 2000 & $15.5 \pm 2.3$ & 453 & 1427 \\
\hline
\end{tabular}

LABOCA map traces about $40 \%$ of the total molecular cloud emission, a fraction that appears very reasonable considering the arguments about the unrecovered large-scale sub-mm flux given above.

\subsection{The spectral energy distribution of the complex}

A good way to study the global energetics and properties of the CNC is to analyze the global spectral energy distribution (SED) of the complex. Smith \& Brooks (2007) constructed the SED in the wavelength range from $8 \mu \mathrm{m}$ to $100 \mu \mathrm{m}$ from MSX and IRAS data (excluding the flux of the star $\eta$ Car) and modeled these data (their Fig. 2) with a combination of three different optically thin graybody components with discrete temperatures of $220 \mathrm{~K}, 80 \mathrm{~K}$, and $35 \mathrm{~K}$. They used this model to estimate the total dust masses associated to these components and suggested that the $\mathrm{CNC}$ contains about $10^{6} M_{\odot}$ of gas; they claimed that there is about three times more gas in atomic form than molecular gas. However, they also noted that their fit is not unique; equally good fits can be obtained with different sets of temperature components, and thus these mass estimates are uncertain. 
Our LABOCA data provide a very important new SED point at longer wavelengths, which strongly constrains possible models of the SED and allows us to obtain new insights into the mass budget of the complex. As mentioned above, the total integrated flux (above the $3 \sigma$ noise limit) in our LABOCA map is $1147 \mathrm{Jy}$. Subtracting the flux from $\eta$ Car (43 Jy), to be consistent with the analysis of SB07, the total flux from the complex is $1100 \mathrm{Jy}$. This value is nearly 20 times smaller than the $870 \mu \mathrm{m}$ flux predicted by the the model of SB07 (see their Fig. 2). Even if we take into account that the field of our LABOCA map is considerably smaller than the $\simeq 5.6$ square-degree area for which the SED points in SB07 were determined ${ }^{7}$, a large discrepancy of a factor of $\sim 14$ remains.

Several possible effects could contribute to this discrepancy. First, the choice of the discrete temperatures for the graybody components can strongly affect the predicted sub-mm flux; a slightly higher assumed temperature would reduce the predicted sub-mm flux substantially. A second aspect is the wavelengthdependence of the dust emissivity: SB07 assumed an emissivity $\propto \lambda^{-1}$, i.e. a dust emissivity index $\beta=1$, which is at the low side of the range of values found by Rathborne et al. (2010) for dark clouds $(\beta=1-2)$. Values of $\beta>1$ would also reduce the predicted sub-mm flux substantially. Thirdly, a three temperature model is obviously a simplification, because the true temperature distribution must be continuous. Considering the comparatively small wavelength range covered by the data that were available for their analysis, the use of a three temperature model by SB07 was quite appropriate, but the availability of our new sub-mm data justify a new modeling attempt.

\subsection{A simple radiative transfer model of the Carina Nebula complex}

In order to investigate the SED in more detail, we performed a simple radiative transfer modeling of the CNC. First, we extracted the fluxes for the SED for the area of our LABOCA map from the $\operatorname{IRAS}^{8}$ and MSX images ${ }^{9}$; the resulting SED is shown in Fig. 9.

Our radiative transfer modeling of the observed SED is not intended to be detailed and highly accurate, but just to see whether we can reproduce the general shape of the observed SED with reasonable assumptions about the mass and largescale density distribution of the surrounding clouds. We do not intend to model the small-scale structure of the individual clouds; instead, we simply assume a central source of radiation

\footnotetext{
7 We analyzed the $100 \mu \mathrm{m}$ IRAS map (since the luminosity and mass estimates from SB07 are strongly dominated by their assumed $35 \mathrm{~K}$ model component, the amplitude of which is proportional to the $100 \mu \mathrm{m}$ flux) and found that our LABOCA field encloses $\simeq 70 \%$ of the total $100 \mu \mathrm{m}$ flux in the larger field.

8 The total flux in the area of our LABOCA map was found to be $40784 \mathrm{Jy}, 137539 \mathrm{Jy}, 627038 \mathrm{Jy}$, and $832420 \mathrm{Jy}$ for the wavelengths of $12 \mu \mathrm{m}, 25 \mu \mathrm{m}, 60 \mu \mathrm{m}$, and $100 \mu \mathrm{m}$, respectively.

9 In order to convert the MSX fluxes, which are given in units of radiance, to intensity units at the isophotal wavelength, we used the conversion factors listed in the General Description of MSX Images from the NASA/IPAC Infrared Science Archive (see http://irsa.ipac.caltech.edu/applications/MSX/MSX/ imageDescriptions .htm). For band A, we used an additional correction factor of 2, as recommended for blackbodies and modified blackbodies at temperatures of about $120-140 \mathrm{~K}$. The corresponding correction factors for the other bands are very close to unity. The resulting fluxes are $10211 \mathrm{Jy}, 57784 \mathrm{Jy}, 55200 \mathrm{Jy}$, and $148479 \mathrm{Jy}$ for the $8 \mu \mathrm{m}$, $12 \mu \mathrm{m}, 14 \mu \mathrm{m}$, and $21 \mu \mathrm{m}$ band, respectively.
}

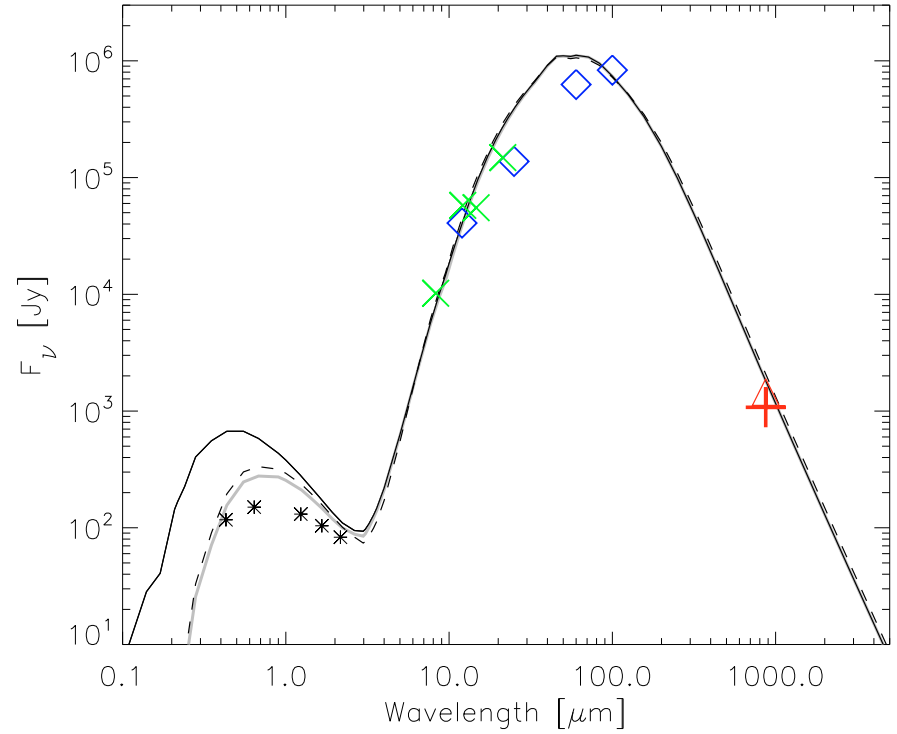

Fig. 9. Spectral energy distribution of the Carina Nebula complex. The diamonds and crosses show the mid- and far-infrared fluxes determined from the IRAS and MSX maps for the field of the LABOCA map. The cross with the upward triangle shows the total $870 \mu \mathrm{m}$ flux derived from our LABOCA map (excluding the emission from $\eta$ Car and the Keyhole Nebula). The asterisks show the integrated optical and nearinfrared fluxes of the known stellar members of Carina as identified in a deep Chandra X-ray survey (see Preibisch et al. 2011b). The solid line shows the spectral energy distribution resulting from our spherical cloud model of the $\mathrm{CNC}$ with a total gas + dust mass of $140000 M_{\odot}$; the gray line shows the effect of adding 1 mag of visual foreground extinction to the spectrum. The dashed line is the model with a cloud mass of $280000 M_{\odot}$.

surrounded by a spherical envelope of dust and gas. Although this is obviously a strong simplification, it provides the advantage that the temperature distribution of the gas is computed in a more physically meaningful way than adding up a few discrete graybody components.

The radiation transfer calculations were carried out with a modified version of the code of Yorke (1980a,b). This program yields an exact solution of the frequency-dependent radiation transfer problem in spherical geometry simultaneously with a self-consistent determination of the dust temperatures. One has to specify the luminosity and the effective temperature of the central source, as well as the density distribution of the surrounding cloud and can compute the overall spectrum and the flux distribution at each wavelength. We use a grid 64 different wavelength points ${ }^{10}$ ranging from $0.1 \mu \mathrm{m}$ to $5 \mathrm{~mm}$. The dust model from Preibisch et al. (1993) was employed, which had been constructed to match the dust properties in molecular clouds. It consists of small $(7-0 \mathrm{~nm})$ amorphous carbon grains and large $(40 \mathrm{~nm}-1 \mu \mathrm{m})$ silicate grains, and assumes that the silicate grains are coated with a thin mantle (thickness $=14.5 \%$ of the grain radius) of "dirty ice" if their temperature is below $125 \mathrm{~K}$. The inner and outer edges of the spatial grid are at $r=10^{16} \mathrm{~cm}$ $(0.003 \mathrm{pc})$ and $28 \mathrm{pc}$.

Following the census of massive stars in the CNC by Smith (2006), we assumed a total stellar luminosity of $2.4 \times 10^{7} L_{\odot}$ and a typical temperature of $T_{\text {eff }}=44700 \mathrm{~K}$. In the first series of models, we set the total (gas + dust) mass of the cloud to

10 The wavelength grid has a approximately equidistant steps in $\log \lambda$ and includes most optical to far-infrared standard bands. 
$140000 M_{\odot}$, following the CO mass estimate of Yonekura et al. (2005). The free parameters in our modeling were the characteristics of the radial density distribution of the cloud. The model shown in Fig. 9 assumes that the density slowly increases with distance from the center according to $\rho(r) \propto r^{0.75}$; this should approximately match the conditions in the $\mathrm{CNC}$, where most of the cloud material has already been dispersed from the central region and is now located at typical distances between $\sim 5 \mathrm{pc}$ and $\sim 25 \mathrm{pc}$.

The computed dust temperatures in this model range from $144 \mathrm{~K}$ (carbon grains) and $69 \mathrm{~K}$ (silicate grains) at $5 \mathrm{pc}$, over $103 \mathrm{~K}$ and $51 \mathrm{~K}$ at $10 \mathrm{pc}$, to $34 \mathrm{~K}$ and $23 \mathrm{~K}$ at $28 \mathrm{pc}$ distance from the central luminosity source. The spectrum of this simple model matches the observed mid-infrared to sub-mm fluxes not perfectly, but reasonably well. The predicted $100 \mu \mathrm{m}$ flux is $739299 \mathrm{Jy}$, i.e. $11 \%$ lower than the value extracted from the IRAS image. The predicted $870 \mu \mathrm{m}$ flux is $1816 \mathrm{Jy}$, i.e. $\approx 70 \%$ higher than the observed LABOCA flux of $1077 \mathrm{Jy}$. This difference agrees well with the expected level of missing large-scale flux in the LABOCA map estimated above from the CO masses. Assuming the missing flux of 729 Jy to be evenly distributed over the area of our LABOCA map would require an unrecovered homogenous flux level of only $\approx 0.014 \mathrm{Jy} / \mathrm{beam}$.

The most important characteristics of this model is that it reproduces both far-infrared and sub-mm fluxes reasonably well. There is therefore no need to postulate a large mass component of widely distributed purely atomic gas that would be neither detectable in the $\mathrm{CO}$ maps nor in the sub-mm map. All available data, i.e. the far-infrared fluxes, the sub-mm flux, and the results derived from the $\mathrm{CO}$ maps, can be explained by a total gas and dust mass of about $140000 M_{\odot}$. We note that this number also agrees well with the recent results from hydrodynamical modeling of the Carina super-bubble by Harper-Clark \& Murray (2009), who derived a total (dust + gas) mass of the nebula of $\sim 10^{5} M_{\odot}$. Finally, it is interesting to note that adding $1.0 \mathrm{mag}$ of visual foreground extinction yields quite good agreement between the model spectrum and the observed integrated optical and near-infrared fluxes of the stellar member of the CNC.

In a second series of models we investigated the effect of increasing the total gas mass above the $140000 M_{\odot}$ of gas as traced by $\mathrm{CO}$ emission. In particular, we wanted to find out whether adding significant amounts of (atomic) gas would raise the $100 \mu \mathrm{m}$ flux to the observed level. We found this not to be the case. Increasing the total gas mass actually leads to lower $100 \mu \mathrm{m}$ model fluxes, because the optical thickness of the gas envelope increases, which leads to cooler dust temperatures in the outer parts of the nebula. This effect can be partly compensated for by assuming a steeper outward density increase. In the second model shown in Fig. 9 we assumed a total gas mass of $280000 M_{\odot}$, i.e. twice the mass as traced by CO. With a density law $\rho(r) \propto r$ we can find an acceptable SED fit. The predicted $100 \mu \mathrm{m}$ flux of this model is $722721 \mathrm{Jy}, 13 \%$ lower than observed. The $870 \mu \mathrm{m}$ flux is $2076 \mathrm{Jy}$, only $14 \%$ higher than in the lower mass model; this small increase in sub-mm flux despite the higher mass is caused by the cooler dust temperatures in the outer parts of this model $(25 \mathrm{~K}$ and $22 \mathrm{~K}$ for the carbon and silicate grains at the outer edge of the model grid).

\subsection{Star formation in the observed clouds}

The dense clouds seen by LABOCA are the site of the current and future star-formation activity in the CNC. Recent observations have revealed hundreds to thousands of very young ( $\lesssim 1 \mathrm{Myr}$ old) stellar objects in these clouds throughout the CNC
(Smith et al. 2010a,b). With our new results on the mass budget of the clouds, we can address the question of how many additional stars may form in the near future in the CNC.

\subsubsection{Cloud mass above the star-formation threshold}

From studies of other molecular cloud complexes it is well known that typically only a small fraction of the total cloud mass will form stars, whereas the majority of the mass will finally be dispersed. Only the densest (and coldest) parts of the gas in a typical molecular cloud will be transformed into stars. An estimate of the fraction of the cloud mass available for star formation can be made if the spatial distribution of (column) density in the cloud is known. Froebrich \& Rowles (2010) showed that the extinction (or column density) threshold for star formation in several nearby molecular clouds is typically at $A_{V} \sim 5$ mag, corresponding to a column density threshold of $N_{\mathrm{H}_{2}}=4.7 \times 10^{21} \mathrm{~cm}^{-2}$.

Assuming a typical cloud temperature of $T=20 \mathrm{~K}$, we find from our LABOCA map that the total mass of the clouds above this column density threshold is about $20000 M_{\odot}$. Note that this value agrees quite well with the total mass of all $\mathrm{C}^{18} \mathrm{O}$ clumps as determined by Yonekura et al. (2005). Assuming a total gas mass of $140000-280000 M_{\odot}$ (as derived above), this implies that only a small fraction, $7-14 \%$, of the total gas mass is in a state in which it is available for star formation.

\subsubsection{The possibility of future massive star-formation in the $\mathrm{CNC}$}

An interesting aspect to investigate is whether the current (and future) star-formation process is similar or different from the previous star-formation activity, which created the massive star clusters $\operatorname{Tr} 14,15$, and 16. A particularly important point in this respect is the maximum mass of the forming stars. The clusters $\operatorname{Tr} 14$ and 16 contain numerous very massive $\left(M>50 M_{\odot}\right)$ stars. In the more recently formed embedded population in the South Pillars, however, the most massive star identified so far is the O9.5 V star in the Treasure Chest, which has a comparatively small mass of $\sim 20 M_{\odot}$. This suggests that stars in the newest generation have substantially lower maximum masses than in the older generation. Will this also be true for the stellar populations yet to form in the remaining clouds?

Observations of stellar clusters suggest that there is a relation between the mass $M_{*, \max }$ of the most massive star in a cluster and the total mass $M_{\text {cluster }}$ of all cluster stars (see Weidner et al. 2010). Although it is still debated whether the observed correlation is actually caused by physical processes or instead is an effect of pure random sampling from the IMF in individual star clusters (see Elmegreen 2006), we assume here that the simple empirical relation

$M_{*, \max } \approx 1.2 \times M_{\text {cluster }}^{0.45}$

suggested by Larson (2003) provides a reasonable approximation. Assuming a star-formation efficiency (i.e. the ratio of the total stellar cluster mass versus the original cloud mass) of $S F E<$ 0.3, as established for cluster formation by Lada \& Lada (2003), yields the desired relation between the cloud mass and the maximum mass of the stars that can be expected to form out of this cloud. It suggests that a cloud with a mass of $M_{\text {cloud }} \approx 1000 M_{\odot}$ will yield a maximum stellar mass of $\sim 15 M_{\odot}$, whereas cloud masses of $\geq 12000 M_{\odot}$ are required to form a very massive star $\left(M \geq 50 M_{\odot}\right)$. 
Nearly all cloud clumps we detected in the CNC have masses $\leq 5000 M_{\odot}$, and therefore will according to Eq. (3), yield a maximum stellar mass of $\$ 30 M_{\odot}$. Most clouds, including the infrared dark cloud discussed above, have masses $\leq 1000 M_{\odot}$, and therefore will yield a maximum stellar mass of just $\lesssim 15 M_{\odot}$. Only the cloud complex to the west of $\operatorname{Tr} 14$ is substantially more massive. However, as can be seen in Fig. 8, this cloud with estimated $\sim 15000 M_{\odot}$ is already fragmented into several individual clumps; most likely, each of these individual clumps will form star clusters, and none of them is massive enough to form a very massive $\operatorname{star}\left(M \geq 50 M_{\odot}\right)$, if the assumed relation in Eq. (3) holds.

To summarize, the observed cloud masses suggest that the currently ongoing, presumably triggered (see Smith et al. 2010b) star-formation process will probably not lead to the formation of very massive stars, as present in large numbers in the older, triggering population. This suggests a clear quantitative difference in the formation processes of the currently forming and the earlier generation of stars in the CNC.

\section{Conclusion}

Our wide-field LABOCA map provides the first large-scale survey of the sub-mm emission in the CNC. We find that the cold dust in the complex is distributed in a wide variety of structures, from the very massive $\left(\sim 15000 M_{\odot}\right)$ and dense cloud complex near $\operatorname{Tr} 14$, over several clumps of a few hundred solar masses, to numerous small clumps containing only a few solar masses of gas and dust. Many of the clouds show clear indications that their structure is shaped by the very strong ionizing radiation field and possibly stellar winds.

The total mass of the dense clouds to which LABOCA is sensitive is $\sim 60000 M_{\odot}$. This value agrees fairly well with the mass estimates for the well localized molecular gas traced by ${ }^{13} \mathrm{CO}$. The complex may contain a considerable amount of very widely distributed atomic gas, which is neither recovered in our LABOCA map, nor can be seen in the CO data. Our radiative transfer modeling suggests that the total mass of such an distributed atomic gas component does probably not exceed the total molecular gas mass $\left(140000 M_{\odot}\right)$ in the region. Thus, the total gas mass in the field of our LABOCA map seems to be $\$ 300000 M_{\odot}$.

Only a small fraction $(\sim 10 \%)$ of the gas in the CNC is currently in dense and massive enough clouds to be available for further star formation. Most observed clouds have masses of less than a few $1000 M_{\odot}$; they will most likely not form any very massive stars, as present in large numbers in the older stellar generation in the CNC. This suggests a clear quantitative difference between the currently ongoing process of triggered star-formation and the process that formed the massive cluster $\operatorname{Tr} 14,15$, and 16 a few Myr ago.

It is interesting to compare these results about future star formation in the clouds to the already existing stellar populations. The results of a recent wide-field Chandra X-ray survey of the CNC (Townsley et al. 2011) suggest that the total stellar population of the CNC consists of about 43000 stars with a combined mass of $\sim 27000 M_{\odot}$ (Preibisch et al. 2011b). This value implies that so far $\sim 10 \%$ of the total cloud mass in the complex have been transformed into stars. This fraction is similar to the typical values of the global star-formation efficiency determined for other OB associations (Briceno et al. 2007).

With about $\sim 20000 M_{\odot}$ of dense clouds available for star formation at this moment, the maximum mass of new stars to form within the next $\sim 1$ Myr may be $\$ 7000 M_{\odot}$ if we assume a high star formation efficiency of $30 \%$, but probably $\lesssim 2000 M_{\odot}$ for lower star-formation efficiencies. Compared to the total mass of the already existing stars, this will be a comparatively small addition.

The importance of the ongoing and future star-formation process in the complex may be increased by the effect of stellar feedback: if the irradiation and the winds from the massive stars efficiently compress the clouds, and continuously transform part of the low-density clouds into denser clouds, the cloud mass available for star formation could well increase. However, it is unlikely that this effect can significantly change the ratio between the stellar populations, simply because time is running out: within less than $\sim 1 \mathrm{Myr}, \eta$ Car will explode as a supernovae. This event will be followed by series of $\sim 70$ further supernova explosions from the massive stars in the complex (see Smith \& Brooks 2008). Each of these explosions will send strong shockwaves through the clouds. While supernova shockwaves are very destructive for any interstellar material in their immediate surroundings, they decay into much slower and weaker shocks after traveling distances of $\gtrsim 1 \mathrm{pc}$. Today, most of the molecular clouds in the CNC are already located at the periphery of the complex, typically a few pc away from the massive stars; these clouds will then be compressed, but probably not destroyed by the crossing "evolved" shockwaves. At locations where suitable conditions are met (see, e.g., Vanhala \& Cameron 1998; Oey \& Garcia-Segura 2004), vigorous star formation activity can then be expected (see, e.g., Preibisch \& Zinnecker 2007, for the example of the Scorpius-Centaurus Association). These supernova shockwaves can not only trigger star formation but also inject short-lived radionucleids such as ${ }^{60} \mathrm{Fe}$ into the collapsing protostellar clouds (Boss \& Keiser 2010). Because there is strong evidence that such short-lived radionucleids were incorporated into the solar nebula material during the formation of our solar system, the clouds in the CNC may provide a good template in which to study the initial conditions for the formation of our solar system.

Acknowledgements. We would like to thank the referee for insightful comments that helped to improve the paper. We gratefully acknowledge funding of this work by the German Deutsche Forschungsgemeinschaft, DFG project number PR 569/9-1. Additional support came from funds from the Munich Cluster of Excellence: "Origin and Structure of the Universe". This publication makes use of data products from the Two Micron All Sky Survey, which is a joint project of the University of Massachusetts and the Infrared Processing and Analysis Center/California Institute of Technology, funded by the National Aeronautics and Space Administration and the National Science Foundation. This work makes use of observations made with the Spitzer Space Telescope, which is operated by the Jet Propulsion Laboratory, California Institute of Technology under a contract with NASA. The reduction of the HST image used in our analysis was supported by STScI Archival Research proposal 11765 (PI Mutchler) and the Hubble Heritage project.

\section{References}

Beaumont, C. N., \& Williams, J. P. 2010, ApJ, 709, 791

Boss, A. P., \& Keiser, S. A. 2010, ApJ, 717, L1

Briceno, C., Preibisch, Th., Sherry, W., et al. 2007, in Protostars \& Planets V, ed.

B. Reipurth, D. Jewitt, \& K. Keil (Tucson: University of Arizona Press), 345 Brooks, K. J., Whiteoak, J. B., \& Storey, J. W. V. 1998, PASA, 15, 202

Brooks, K. J., Cox, P., Schneider, N., et al. 2003, A\&A, 412, 751

Brooks, K. J., Garay, G., Nielbock, M., Smith, N., \& Cox, P. 2005, ApJ, 634, 436

Carey, S. J., Clark, F. O., Egan, M. P., et al. 1998, ApJ, 508, 721

Cox, P., \& Bronfman, L. 1995, A\&A, 299, 583

Dale, J. E., \& Bonnell, I. A. 2008, MNRAS, 391, 2

Dale, J. E., Bonnell, I. A., Clarke, C. J., \& Bate, M. R. 2005, MNRAS, 358, 291

Deharveng, L., Zavagno, A., Schuller, F., et al. 2009, A\&A, 496, 177

Elmegreen, B. G. 2006, ApJ, 648, 572 
Freyer, T., Hensler, G., \& Yorke, H. W. 2003, ApJ, 594, 888

Froebrich, D., \& Rowles, J. 2010, MNRAS, 406, 1350

Gomez, H. L., Vlahakis, C., Stretch, C. M., et al. 2010, MNRAS, 401, L48

Gritschneder, M., Naab, Th., Walch, S., Burkert, A., \& Heitsch, F. 2009, ApJ, 694, L26

Groh, J. H., Madura, T. I., Owocki, S. P., Hillier, D. J., \& Weigelt, G. 2010, ApJ, 716, L223

Güsten, R., Nyman, L. Å., Schilke, P., et al. 2006, A\&A, 454, L13

Harper-Clark, E., \& Murray, N. 2009, ApJ, 693, 1696

Henning, T., Michel, B., \& Stognienko, R. 1995, Planet. Space Sci., 43, 1333

Hildebrand, R. H. 1983, QJRAS, 24, 267

Kramer, C., Cubick, M., Röllig, M., et al. 2008, A\&A, 477, 547

Lada, C. J., \& Lada, E. A. 2003, ARA\&A, 41, 57

Larson, R. B. 2003, in Galactic Star Formation Across the Stellar Mass Spectrum, ASP Conf. Ser., 287, 65

Maruta, H., Nakamura, F., Nishi, R., Ikeda, N., \& Kitamura, Y. 2010, ApJ, 714, 680

Megeath, S. T., Cox, P., Bronfman, L., \& Roelfsema, P. R. 1996, A\&A, 305, 296

Mehner, A., Davidson, K., Humphreys, R. M., et al. 2010, ApJ, 717, L22

Mutchler, M., \& Bowers, A. 2009, http://archive.stsci.edu/prepds/ carina/

Oey, M. S., \& Garcia-Segura, G. 2004, ApJ, 613, 302

Ossenkopf, V., \& Henning, T. 1994, A\&A, 291, 943

Preibisch, Th., \& Zinnecker, H. 2007, IAU Symp., 237, 270

Preibisch, Th., Ossenkopf, V., Yorke, H. W., \& Henning, T. 1993, A\&A, 279, 577
Preibisch, Th., Hodgkin, S., Irwin, M., et al. 2011b, ApJS, submitted Rathborne, J. M., Jackson, J. M., \& Simon, R. 2006, ApJ, 641, 389

Rathborne, J. M.. Jackson, J. M.. Chambers, E. T., et al. 2010, ApJ, 715, 310

Rygl, K. L. J., Wyrowski, F., Schuller, F., \& Menten, K. M. 2010, A\&A, 515, A42

Sana, H., Momany, Y., Gieles, M., et al. 2010, A\&A, 515, A26

Schneider, N., \& Brooks, K. 2004, PASA, 21, 290

Schuller, F., Menten, K. M., Contreras, Y., et al. 2009, A\&A, 504, 415

Siringo, G., et al. 2009, A\&A, 497, 945

Smith, N. 2006, MNRAS, 367, 763

Smith, N., \& Brooks, K. J. 2007, MNRAS, 379, 1279

Smith, N., \& Brooks, K. J. 2008, Handbook of Star Forming Regions, Vol. II: The Southern Sky, ed. B. Reipurth, ASP Monograph Publ., 5, 138

Smith, N., Stassun, K. G., \& Bally, J. 2005, AJ, 129, 888

Smith, N., Bally, J., \& Walborn, N. R. 2010a, MNRAS, 405, 1153

Smith, N., Povich, N. S., Whitney, B. A., et al. 2010b, MNRAS, 406, 952

Stamatellos, D., Whitworth, A. P., \& Ward-Thompson, D. 2007, MNRAS, 379, 1390

Tapia, M., Roth, M., Vazques, R. A., \& Feinstein, A. 2003, MNRAS, 339, 444

Townsley, L., Broos, P. S., Corcoran, M. F., et al. 2011, ApJS, submitted

Trumpler, R. J. 1930, Lick Observatory Bulletin, 14, 154

Vanhala, H. A. T., \& Cameron, A. G. W. 1998, ApJ, 508, 291

Weidner, C., Kroupa, P., \& Bonnell, I. A. D. 2010, MNRAS, 401, 275

Yonekura, Y., Asayama, S., Kimura, K., et al. 2005, ApJ 634, 476

Yorke, H. W. 1980a, A\&A, 85, 215

Yorke, H. W. 1980b, A\&A, 86, 286 